\title{
A Stable and Convergent Scheme for Viscoelastic Flow in Contraction Channels
}

\author{
D. Trebotich* \\ Center for Applied Scientific Computing, Lawrence Livermore National \\ Laboratory, P.O. Box 808, L-560, Livermore, CA 94551, USA \\ P. Colella \\ Applied Numerical Algorithms Group, Lawrence Berkeley National Laboratory, \\ 1 Cyclotron Road, Berkeley, CA 94720, USA \\ G. H. Miller \\ Department of Applied Science, University of California, One Shields Avenue, \\ Davis, CA 95616, USA
}

\begin{abstract}
We present a new algorithm to simulate unsteady viscoelastic flows in abrupt contraction channels. In our approach we split the viscoelastic terms of the Oldroyd-B constitutive equation using Duhamel's formula and discretize the resulting PDEs using a semi-implicit finite difference method based on a Lax-Wendroff method for hyperbolic terms. In particular, we leave a small residual elastic term in the viscous limit by design to make the hyperbolic piece well-posed. A projection method is used to impose the incompressibility constraint. We are able to compute the full range of elastic flows in an abrupt contraction channel - from the viscous limit to the elastic limit - in a stable and convergent manner for elastic Mach numbers less than one. We demonstrate the method for unsteady Oldroyd-B and Maxwell fluids in planar contraction channels.
\end{abstract}

Key words: viscoelastic flow, Oldroyd-B fluid, Maxwell fluid, projection methods, contraction channels

PACS: 65N06, 76D05

\footnotetext{
* Corresponding author. Email addresses: trebotich1@llnl.gov (D. Trebotich), pcolella@lbl.gov (P. Colella), grgmiller@ucdavis.edu (G. H. Miller).
} 


\section{Introduction}

In this paper, we present a numerical method for solving the equations describing incompressible viscoelastic fluids such as dilute polymer solutions

$$
\begin{aligned}
\rho \frac{\partial \mathbf{u}}{\partial t}+\rho(\mathbf{u} \cdot \nabla) \mathbf{u} & =-\nabla p+\mu_{s} \Delta \mathbf{u}+\nabla \cdot \boldsymbol{\tau} \\
\nabla \cdot \mathbf{u} & =0 .
\end{aligned}
$$

Here, $\mu_{s}$ is the solvent viscosity, and the non-Newtonian contribution of the stress is given by the Oldroyd-B model

$$
\boldsymbol{\tau}+\lambda \stackrel{\nabla}{\boldsymbol{\tau}}=2 \mu_{p} \mathbf{D}
$$

where $\stackrel{\nabla}{\tau}$ is the upper-convected Maxwell (UCM) derivative (see Eq. 24 below). If $\mu_{s}$ vanishes, then this system is often referred to as the Maxwell model. We choose the Oldroyd-B model as it is sufficiently well-understood to serve as a test bed for numerical methods.

Our approach is based on the method of Bell, Colella and Glaz (BCG) [3], a second-order accurate version of Chorin's projection method [5]. We use a continuous splitting of the relaxation terms so that, in the limit of vanishing $\lambda$, we recover a BCG-type algorithm for a Newtonian fluid with an augmented viscosity. In particular, the time step is controlled only by the advective CFL number in that limit. In the elastic limit $\lambda \rightarrow \infty, \mu_{p} / \lambda$ finite, we obtain a method whose time step is controlled only by the CFL condition for the elastic shear waves. Kupferman employed a BCG-type projection method with staggered centering to study stability of viscoelastic fluids in Taylor-Couette flow [14]. Our method differs from the one presented there in that (1) in general, we consider high $W e$ flows in the presence of geometric singularities; (2) our method uses a much less restrictive time step in the viscous limit; and (3) we use fixed cell centering which is more amenable to the approaches taken in [2] for adaptive mesh refinement and [10] for embedded boundaries.

Numerical simulation of viscoelastic flow in contraction channels (Figure 1) is a problem that has been given much attention over the past 30 years because of its importance to polymer processing $[7,6]$. Much has been published on the inability to compute strongly-elastic, "high Weissenberg number" flows ones for which the ratio of the elastic relaxation time scale to the advective time scale is large - particularly in the presence of geometric singularities such as re-entrant corners. These limits have been observed primarily in the context of numerical simulations of steady flows. Keunings [13] used a finiteelement method to compute steady 2D flows of Maxwell fluids through 4:1 
planar sudden contractions. The observed critical values of $W e$ were less than one (0.112 to 0.873 , depending on the mesh). Lipscomb et al. [17] repeated the Keunings calculations with the single change - the relaxation time was set to zero in the re-entrant corner element to control the stress singularity - increasing the critical We from 0.1 to 0.6 with no noticeable difference in flow outside of the corner element. Many attempts followed, of which a detailed literature review is given by Phillips and Williams [19] who used a semi-Lagrangian finite volume method to observe steady-state solutions for both creeping and inertial flows in planar contractions, obtaining results up to $W e=2.5$. They also clarify definitions of Weissenberg and Deborah $(D e)$ numbers, useful for comparing high $W e$ to previous studies in high $D e$ as in [18]. Xue, Phan-Thien and Tanner [26] presented a finite volume method for both $2 \mathrm{D}$ and $3 \mathrm{D}$ problems and observed a critical value of $W e=4.4$. Recently, Aboubacar et al. [1] employed a hybrid finite volume/finite element scheme for Oldroyd-B creeping flows in rounded and abrupt contractions, and observed a critical value of $W e=4.6$.

The high Weissenberg number problem is actually a frustratingly low Weissenberg number problem for steady flows beyond which stable and convergent calculations have not been achievable. There appears to be no analytic expression for the limiting value of the Weissenberg number, and considerable indication that the critical value depends on the mesh resolution. One of the principal conclusions of this paper is that the difficulties that have been observed in the prior work may be an artifact of using steady-state or implicit methods, rather than an appropriately-designed unsteady flow method that can resolve the hyperbolic wave behavior of the problem. This is consistent with the point of view taken in $[25,12]$, in which the elastic Mach number - the ratio of the fluid velocity to the elastic wave velocity - is identified as the critical parameter in understanding viscoelastic flows. In particular, we find that our method is stable and accurate for any value of the Weissenberg number, provided that the elastic Mach number is less than one. This restriction on the elastic Mach number may be a consequence of the use of a Lax-Wendroff discretization for the hyperbolic terms, rather than a welldesigned upwind method suitable for use in situations when discontinuities might spontaneously appear. We will explore this possibility in future work.

A preliminary version of these results appears in [23].

\section{Analysis of PDEs}

If we define the Cauchy stress by splitting the extra stress into a solvent and polymer parts 


$$
\mathbf{T}=-p \mathbf{I}+2 \mu_{s} \mathbf{D}+\boldsymbol{\tau}
$$

then we can write the equations of motion as

$$
\begin{aligned}
\rho \frac{\partial \mathbf{u}}{\partial t}+\rho(\mathbf{u} \cdot \nabla) \mathbf{u}+\nabla p & =\mu_{s} \Delta \mathbf{u}+\nabla \cdot \boldsymbol{\tau} \\
\nabla \cdot \mathbf{u} & =0 \\
\frac{\partial \boldsymbol{\tau}}{\partial t}+(\mathbf{u} \cdot \nabla) \boldsymbol{\tau}-\nabla \mathbf{u} \cdot \boldsymbol{\tau}-\boldsymbol{\tau} \cdot \nabla \mathbf{u}^{T} & =\frac{1}{\lambda}\left[\mu_{p}\left(\nabla \mathbf{u}+\nabla \mathbf{u}^{T}\right)-\boldsymbol{\tau}\right]
\end{aligned}
$$

with $\mathbf{u}=(u, v, w)$ the solution velocity, $\boldsymbol{\tau}$ the polymer stress, $\lambda$ the relaxation time, $\mu_{s}$ the dynamic viscosity of the solvent, $\mu_{p}$ the dynamic viscosity of the polymer, and $\rho$ the density of the aqueous solution (approximately that of water). We also define two useful dimensionless measures relating elasticity to the fluid. The Weissenberg number $[4,11,13]$ is the ratio of the polymer relaxation time to the advective time scale

$$
W e=\frac{\lambda U}{L}
$$

where $U$ is the characteristic velocity of the flow, usually the average fluid velocity in the contracted channel, and $L$ is the characteristic length scale of the flow, usually half the width of the small channel in contraction flows. The elastic Mach number $[25,12]$ is the ratio of the advective wavespeed to the elastic wavespeed

$$
M a=\frac{U}{\sqrt{\mu_{p} / \rho \lambda}}
$$

Continuum mechanics and applied mathematics conventions differ for the compact notation used in (7). Here we use the convention that $(\nabla \mathbf{u})_{\alpha \beta}=$ $(\partial \mathbf{u} / \partial \mathbf{x})_{\alpha \beta}=\partial \mathbf{u}_{\alpha} / \partial \mathbf{x}_{\beta}$, whence (7) is equivalent to

$$
\frac{\partial \boldsymbol{\tau}_{\alpha \beta}}{\partial t}+\mathbf{u}_{\gamma} \frac{\partial \boldsymbol{\tau}_{\alpha \beta}}{\partial \mathbf{x}_{\gamma}}-\frac{\partial \mathbf{u}_{\alpha}}{\partial x_{\gamma}} \boldsymbol{\tau}_{\gamma \beta}-\boldsymbol{\tau}_{\alpha \gamma} \frac{\partial \mathbf{u}_{\beta}}{\partial \mathbf{x}_{\gamma}}=\frac{1}{\lambda}\left[\mu_{p}\left(\frac{\partial \mathbf{u}_{\alpha}}{\partial \mathbf{x}_{\beta}}+\frac{\partial \mathbf{u}_{\beta}}{\partial \mathbf{x}_{\alpha}}\right)-\boldsymbol{\tau}_{\alpha \beta}\right](10)
$$

The momentum equation (5) is an advection-diffusion type with a source term and is well understood in the context of our numerical approach. The stress equation (7) at first glance is of similar type; however, we note two important limits that affect the equation type: (i) the viscous limit where $\lambda=0$, and (ii) the elastic limit where $\lambda \rightarrow \infty, \mu_{p} / \lambda$ finite. Therefore, we would like to manipulate (7) into a form which exploits its hyperbolicity for coupling to (5) 
in the context of a BCG-type approach, while at the same time capturing the viscous and elastic limits in a seemless and stable manner.

Boundary conditions are needed to completely specify the problem. Velocity boundary conditions are as follows: (1) no-slip, no-flow solid walls, $\mathbf{u}=0 ;(2)$ prescribed plug flow at inflow; and (3) no gradients in normal direction at outflow, $\frac{\partial \mathbf{u}}{\partial n}=0$.

\subsection{Linearized Equations}

We begin the system analysis by linearizing the equations in order to cast them in a hyperbolic form. First, we consider the homogeneous part of (5) expanded in 1D (direction 1 or $\hat{x}$ )

$$
\frac{\partial u_{i}}{\partial t}+u_{1} \cdot \frac{\partial u_{i}}{\partial x_{1}}-\frac{1}{\rho} \frac{\partial \tau_{1 i}}{\partial x_{1}}=0
$$

Similarly, with the stress equation (7), we expand the homogeneous part in $1 \mathrm{D}$ to obtain

$$
\frac{\partial \tau_{1 j}}{\partial t}+u_{1} \frac{\partial \tau_{1 j}}{\partial x_{1}}-\frac{\partial u_{1}}{\partial x_{1}} \tau_{1 j}-\tau_{11} \frac{\partial u_{j}}{\partial x_{1}}-\frac{\mu_{p}}{\lambda} \frac{\partial u_{1}}{\partial x_{1}} \delta_{1 j}-\frac{\mu_{p}}{\lambda} \frac{\partial u_{j}}{\partial x_{1}}=0
$$

The linearized system of equations is then

$$
\frac{\partial \mathbf{U}}{\partial t}+\mathbf{A} \frac{\partial \mathbf{U}}{\partial x_{1}}=0
$$

where

$$
\mathbf{U}=\left(\begin{array}{c}
u_{1} \\
u_{2} \\
u_{3} \\
\tau_{11} \\
\tau_{12} \\
\tau_{13}
\end{array}\right)
$$

and 


$$
\mathbf{A}=\left(\begin{array}{cccccc}
u_{1} & 0 & 0 & -\frac{1}{\rho} & 0 & 0 \\
0 & u_{1} & 0 & 0 & -\frac{1}{\rho} & 0 \\
0 & 0 & u_{1} & 0 & 0 & -\frac{1}{\rho} \\
-2 \tau_{11}-2 \frac{\mu_{p}}{\lambda} & 0 & 0 & u_{1} & 0 & 0 \\
-\tau_{12} & -\tau_{11}-\frac{\mu_{p}}{\lambda} & 0 & 0 & u_{1} & 0 \\
-\tau_{13} & 0 & -\tau_{11}-\frac{\mu_{p}}{\lambda} & 0 & 0 & u_{1}
\end{array}\right) .
$$

The eigenvalues $\gamma, \zeta$ and eigenvectors $\boldsymbol{\xi}, \boldsymbol{\chi}$ can now be obtained for this system. The transverse modes are doubly degenerate

$$
\gamma_{ \pm}=u_{1} \pm \sqrt{\left(\mu_{p} / \lambda+\tau_{11}\right) / \rho},
$$

with eigenvectors

$$
\boldsymbol{\xi}_{ \pm}^{+}=\left(\begin{array}{c}
0 \\
0 \\
\mp 1 \\
0 \\
0 \\
\sqrt{\rho\left(\mu_{p} / \lambda+\tau_{11}\right)}
\end{array}\right) \text {, and } \boldsymbol{\xi}_{ \pm}^{-}=\left(\begin{array}{c}
0 \\
\mp 1 \\
0 \\
0 \\
\sqrt{\rho\left(\mu_{p} / \lambda+\tau_{11}\right)} \\
0
\end{array}\right) .
$$

The longitudinal modes are

$$
\zeta_{ \pm}=u_{1} \pm \sqrt{2\left(\mu_{p} / \lambda+\tau_{11}\right) / \rho}
$$

with eigenvectors

$$
\boldsymbol{\chi}_{ \pm}=\left(\begin{array}{c}
\mp\left(\mu_{p} / \lambda+\tau_{11}\right) \sqrt{2} \\
\mp \tau_{12} \sqrt{2} \\
\mp \tau_{13} \sqrt{2} \\
2\left(\mu_{p} / \lambda+\tau_{11}\right) \sqrt{\rho\left(\mu_{p} / \lambda+\tau_{11}\right)} \\
2 \tau_{12} \sqrt{\rho\left(\mu_{p} / \lambda+\tau_{11}\right)} \\
2 \tau_{13} \sqrt{\rho\left(\mu_{p} / \lambda+\tau_{11}\right)}
\end{array}\right) .
$$

However, we note that for incompressible flow, the longitudinal modes cannot appear, since plane waves of this type would violate the incompressibility constraint.

According to $(16,18)$ the system is hyperbolic as long as

$$
\tau_{11}>-\frac{\mu_{p}}{\lambda} .
$$




\subsection{Proof of Hyperbolicity}

A proof that the homogeneous PDEs are hyperbolic (see also Rutkevich [21,22], Crochet [6] and Joseph $[11, \S 1.16])$ makes reference to the deformation gradient

$$
\mathbf{F}=\frac{\partial \mathbf{x}}{\partial \mathbf{X}}
$$

relating a spatial (Eulerian) coordinate system $\{\mathbf{x}\}$ to a material (Lagrangian) coordinate system $\{\mathbf{X}\}$. The Jacobian $J=\operatorname{det}(\mathbf{F})$ is the ratio of material specific volume $(1 / \rho)$ to specific volume in the material reference frame. Since $J$ is strictly positive, the inverse of $\mathbf{F}$ exists:

$$
\mathbf{g}=\mathbf{F}^{-1}
$$

is the inverse deformation gradient. The symmetric tensor $\operatorname{gg}^{T}$ is positive definite.

The velocity gradient may be written in terms of the material derivative $\left(\frac{d}{d t}\right)$ of the deformation gradient:

$$
\frac{\partial u_{\alpha}}{\partial x_{\beta}}=\frac{d F_{\alpha \gamma}}{d t} g_{\gamma \beta} .
$$

Using this result, the upper-convected Maxwell derivative of any tensor $\mathbf{H}$,

$$
\stackrel{\nabla}{\mathbf{H}} \equiv \frac{d \mathbf{H}}{d t}-(\nabla u) \mathbf{H}-\mathbf{H}(\nabla u)^{T}
$$

may be written

$$
\stackrel{\nabla}{\mathbf{g H g}^{T}}=\frac{d}{d t}\left(\mathbf{g H g}^{T}\right)
$$

The stress equation (7) may therefore be written

$$
\frac{d}{d t}\left(\mathbf{g} \tau \mathbf{g}^{T}\right)+\frac{1}{\lambda} \mathbf{g} \tau \mathbf{g}^{T}=-\frac{\mu_{p}}{\lambda} \frac{d}{d t}\left(\mathbf{g g}^{T}\right)
$$

with solution 


$$
\mathbf{g}\left(\boldsymbol{\tau}+\frac{\mu_{p}}{\lambda} \mathbf{I}\right) \mathbf{g}^{T}=\frac{\mu_{p}}{\lambda^{2}} \int_{-\infty}^{t} d s e^{(s-t) / \lambda} \mathbf{g g}^{T}
$$

Now, since $\mathbf{g g}^{T}$ is positive definite, $\mathbf{g}\left(\boldsymbol{\tau}+\frac{\mu_{p}}{\lambda} \mathbf{I}\right) \mathbf{g}^{T}$ is also positive definite, and so too is $\boldsymbol{\tau}+\frac{\mu_{p}}{\lambda} \mathbf{I}$. The diagonal elements of a positive definite tensor must be positive, so $\tau_{11}+\frac{\mu_{p}}{\lambda}>0$, and therefore the homogeneous PDEs (11) and (12) are hyperbolic.

\section{Design of New Algorithm}

Now that we have shown the hyperbolic nature of the stress equation (7) we can exploit this result to design a new algorithm that makes use of the advantages of a BCG-type projection method while capturing the viscous and elastic limits of the stress equation. We begin by using the previous linear analysis to cast the stress equation (7) in a relatively simple form in terms of the inverse deformation gradient $\mathbf{g}$ :

$$
\frac{d}{d t}\left(\mathbf{g} \tau \mathbf{g}^{T}\right)=-\frac{1}{\lambda}\left[\left(\mathbf{g} \tau \mathbf{g}^{T}\right)+\mu_{p} \frac{d}{d t}\left(\mathbf{g g}^{T}\right)\right]
$$

Integrating (28) from $t^{n}$ to $t^{n+1}=t^{n}+\Delta t$, using Duhamel's formula and the mean value theorem, we obtain

$$
\begin{aligned}
\left(\mathbf{g} \tau \mathbf{g}^{T}\right)^{n+1} & =\left(\mathbf{g} \tau \mathbf{g}^{T}\right)^{n} e^{-\frac{\Delta t}{\lambda}}-\int_{0}^{\Delta t} e^{-\frac{(\Delta t-s)}{\lambda}} \frac{\mu_{p}}{\lambda} \frac{d}{d t}\left(\mathbf{g g}^{T}\right) d s \\
& =\left(\mathbf{g} \tau \mathbf{g}^{T}\right)^{n} e^{-\frac{\Delta t}{\lambda}}+2 \mu_{p} \overline{(\mathbf{g D} \mathbf{g} T)}\left[1-e^{-\Delta t / \lambda}\right]
\end{aligned}
$$

with

$$
\frac{d}{d t}\left(\mathbf{g g}^{T}\right)=2 \mathbf{g D g}^{T}
$$

where $\mathbf{D}=\frac{1}{2}\left[(\nabla \mathbf{u})+(\nabla \mathbf{u})^{T}\right]$ and the overbar denotes the mean value theorem average from 0 to $\Delta t$.

We then recognize the two important limits for our algorithm design. First as the relaxation time approaches zero, $\lambda \rightarrow 0$, the polymer stress equilibrates instantly with the polymer viscous stress:

$$
\boldsymbol{\tau}=\mu_{p}\left(\nabla \mathbf{u}+\nabla \mathbf{u}^{T}\right) \quad(\lambda \rightarrow 0)
$$


In this equilibrium limit, (30) becomes

$$
\left(\mathbf{g} \tau \mathbf{g}^{T}\right)^{n+1}=2 \mu_{p} \mathbf{g} \mathbf{D g}^{T} \quad(\lambda \rightarrow 0)
$$

Second, as the relaxation time increases without bound, the polymer stress equation becomes

$$
\frac{\partial \boldsymbol{\tau}}{\partial t}+\mathbf{u} \cdot \nabla \boldsymbol{\tau}-(\nabla \mathbf{u}) \boldsymbol{\tau}-\boldsymbol{\tau}(\nabla \mathbf{u})^{T}=\frac{\mu_{p}}{\lambda}\left(\nabla \mathbf{u}+\nabla \mathbf{u}^{T}\right) \quad(\lambda \rightarrow \infty)
$$

(Here we require that $\mu_{p} / \lambda$ approaches a definite nonzero limit.) In this fully decoupled limit the integral expression (30) becomes

$$
\left(\mathbf{g} \tau \mathbf{g}^{T}\right)^{n+1}=\left(\mathbf{g} \tau \mathbf{g}^{T}\right)^{n}-\frac{\mu_{p}}{\lambda}\left[\left(\mathbf{g g}^{T}\right)^{n+1}-\left(\mathbf{g g}^{T}\right)^{n}\right] \quad(\lambda \rightarrow \infty)
$$

In order to assure recovery of these limits in a discretization of (28), we introduce a factor $C(\lambda)$ with the following properties:

$$
\begin{aligned}
& \lim _{\lambda \rightarrow 0} C(\lambda)=\mathcal{O}(\lambda) \\
& \lim _{\lambda \rightarrow \infty} C(\lambda)=1 .
\end{aligned}
$$

For example,

$$
C(\lambda)=1-e^{-K \lambda / t_{a d v}}
$$

where $K$ is a positive constant, e.g., $K=1$ or $K=\sigma$, and $\sigma<1$ is the CFL number. Also, we note use of the parameter, $t_{a d v}$, instead of $\Delta t$. This advective time scale on a discrete grid is obtained by applying the advective limit, and, thus, it is defined as the ratio of discrete grid spacing to the maximum fluid velocity,

$$
t_{a d v}=\Delta \mathbf{x} / \max _{i, j, k}|\mathbf{u}|
$$

where $\Delta \mathbf{x}$ corresponds to the grid spacing in the direction of maximum $\mathbf{u}$, at the maximum velocity's discrete cell center $(i, j, k)$. By choosing $t_{a d v}$ to be independent of $\Delta t$, a stable $\Delta t$ can be constructed explicitly from the CFL condition in our discretization. We will also show that this choice of time scale obeys the design limits of the algorithm $(48,47)$.

We may use $C(\lambda)$ to isolate the limiting parts of (30), and rearrange to give 


$$
\begin{aligned}
& \frac{\left(\mathbf{g} \tau \mathbf{g}^{T}\right)^{n+1}-\left(\mathbf{g} \tau \mathbf{g}^{T}\right)^{n}}{\Delta t}=-\left(\mathbf{g} \tau \mathbf{g}^{T}\right)^{n} \frac{\left[1-e^{-\Delta t / \lambda}\right]}{\Delta t} \\
& +2 \mu_{p} \overline{\left(\mathbf{g D g}^{T}\right)} \frac{\left[1-e^{-\Delta t / \lambda}\right]}{\Delta t}[1-C(\lambda)] \\
& +2 \frac{\mu_{p}}{\lambda} \overline{\left(\mathbf{g D g}^{T}\right)} C(\lambda) \lambda \frac{\left[1-e^{-\Delta t / \lambda}\right]}{\Delta t} \text {. }
\end{aligned}
$$

This discretization of (30) recovers the desired limits. As $\lambda \rightarrow 0$,

$$
\frac{\left(\mathbf{g} \tau \mathbf{g}^{T}\right)^{n+1}-\left(\mathbf{g} \tau \mathbf{g}^{T}\right)^{n}}{\Delta t}=-\left(\mathbf{g} \tau \mathbf{g}^{T}\right)^{n} \frac{1}{\Delta t}+2 \mu_{p} \overline{\left(\mathbf{g} \mathbf{D g} \mathbf{g}^{T}\right)} \frac{1}{\Delta t}
$$

and as $\lambda \rightarrow \infty\left(\right.$ with $\left.\lambda\left(1-e^{-\Delta t / \lambda}\right) \rightarrow \Delta t\right)$

$$
\frac{\left(\mathbf{g} \tau \mathbf{g}^{T}\right)^{n+1}-\left(\mathbf{g} \tau \mathbf{g}^{T}\right)^{n}}{\Delta t}=2 \frac{\mu_{p}}{\lambda} \overline{\left.(\mathbf{g D g})^{T}\right)} \underset{\lambda \rightarrow \infty}{=}-\frac{\mu_{p}}{\lambda} \frac{\left(\mathbf{g g}^{T}\right)^{n+1}-\left(\mathbf{g g}^{T}\right)^{n}}{\Delta t}
$$

Let us associate the part surviving $\lambda \rightarrow \infty$ with an explicit hyperbolic scheme having the form

$$
\frac{\partial}{\partial t}\left(\mathbf{g} \tau \mathbf{g}^{T}\right)-2 a^{2}\left(\mathbf{g D g} \mathbf{g}^{T}\right)=\ldots
$$

This choice is motivated by the fact that in the fully uncoupled limit (34) may be written as a conservation law accurately modeled with higher-order advection schemes:

$$
\frac{d}{d t} \mathbf{g}\left(\boldsymbol{\tau}+\frac{\mu_{p}}{\lambda} \mathbf{I}\right) \mathbf{g}^{T}=0
$$

By comparing (43) with (40) we define a wavespeed

$$
\begin{aligned}
a^{2} & =\alpha(\lambda) a_{\infty}^{2}+[1-\alpha(\lambda)] a_{0}^{2} \\
\alpha(\lambda) & =\frac{\lambda}{\Delta t} C(\lambda)\left[1-e^{-\Delta t / \lambda}\right] \\
a_{\infty}^{2} & =\lim _{\lambda \rightarrow \infty} a^{2}=\frac{\mu_{p}}{\lambda}
\end{aligned}
$$

while retaining some elastic freedom in recovering the viscous limit

$$
a_{0}^{2}=\lim _{\lambda \rightarrow 0} a^{2}=\eta \tau .
$$

We define $\tau$ as the absolute value of the global minimum of the normal stresses, $\tau=\left|\min _{\beta} \tau_{\beta \beta}\right|$, and $\eta$ is a positive control parameter usually equal to 1 . 
Also, the remainder, or right-hand side, from comparing (43) with (40) contains a proper source term and gradient viscous terms

$$
-\frac{1}{\lambda}\left[\left(\mathbf{g} \tau \mathbf{g}^{T}\right)-2 \mu_{p}\left(\mathbf{g D g} \mathbf{g}^{T}\right)\right]-2 a^{2}\left(\mathbf{g D} \mathbf{g}^{T}\right)
$$

All together, the stress equation (7) becomes

$$
\begin{array}{r}
\frac{\partial \boldsymbol{\tau}}{\partial t}+\mathbf{u} \cdot \nabla \boldsymbol{\tau}-(\nabla \mathbf{u}) \boldsymbol{\tau}-\boldsymbol{\tau}(\nabla \mathbf{u})^{T}-a^{2}\left[(\nabla \mathbf{u})+\left(\nabla \mathbf{u}^{T}\right)\right]= \\
-\frac{1}{\lambda} \boldsymbol{\tau}+\left[\frac{\mu_{p}}{\lambda}-a^{2}\right]\left[(\nabla \mathbf{u})+(\nabla \mathbf{u})^{T}\right]
\end{array}
$$

From here, our approach is to discretize (50) using an unsplit Lax-Wendroff method which is appropriate for the hyperbolic left-hand side; and an implicit discretization of the right-hand side to recover the viscous limit as $\lambda \rightarrow 0$.

\section{Discretization Method}

We discretize the equations of motion (5), (6) and (50) using a high-resolution finite difference method to evolve velocity, pressure and stress in time. Time is represented in discrete time steps as $t=(n+1) \Delta t, n=0,1,2, \ldots$. At the beginning of each time step we know cell-centered grid representations of velocity, pressure and stress: $\mathbf{u}_{i, j, k}^{n}, \nabla p_{i, j, k}^{n}, \boldsymbol{\tau}_{i, j, k}^{n}$.

\subsection{Velocity Discretization}

We summarize the velocity discretization following ideas in [3]. The projection operator is used to advance the velocity in time while enforcing incompressibility

$$
\begin{aligned}
\mathbf{u}^{n+1} & =\mathbf{u}^{*}-(\mathbf{I}-\mathbf{P})\left(\mathbf{u}^{*}\right) \\
\nabla p^{n+\frac{1}{2}} & =\nabla p^{n-\frac{1}{2}}+\frac{\rho}{\Delta t}(\mathbf{I}-\mathbf{P})\left(\mathbf{u}^{*}\right)
\end{aligned}
$$

where $\mathbf{Q}=\mathbf{G L}^{-1} \mathbf{D}, \mathbf{P}=\mathbf{I}-\mathbf{Q}, \mathbf{L}=\mathbf{D G}$ and $\mathbf{D}$ and $\mathbf{G}$ are numerical divergence and gradient operators, respectively.

To predict the intermediate velocity, $\mathbf{u}^{*}$, we first construct edge-centered velocities using a Lax-Wendroff (LW) discretization of the PDE, omitting the pressure gradient (for now) 


$$
\tilde{\mathbf{u}}_{i+\frac{1}{2}, j, k}=\frac{1}{2}\left(\mathbf{u}_{i, j, k}^{n}+\mathbf{u}_{i+1, j, k}^{n}\right)+\frac{\Delta t}{2}\left(-(\mathbf{u} \cdot \nabla) \mathbf{u}+\frac{\mu_{s}}{\rho} \Delta \mathbf{u}+\frac{1}{\rho} \nabla \cdot \boldsymbol{\tau}\right)_{i+\frac{1}{2}, j, k}^{n}(53)
$$

A projection is then applied to the edge-centered velocities to correct for the omitted pressure term in the previous step

$$
\mathbf{u}_{i+\frac{1}{2}, j, k}^{n+\frac{1}{2}}=\tilde{\mathbf{u}}_{i+\frac{1}{2}, j, k}-\nabla\left(\Delta^{-1}(\nabla \cdot \tilde{\mathbf{u}})\right)_{i+\frac{1}{2}, j, k} .
$$

In this predictor step we use inviscid, no-flow boundary conditions, or $\mathbf{u} \cdot \hat{\mathbf{n}}=0$ at solid walls, $\frac{\partial \mathbf{u}}{\partial \hat{\mathbf{n}}}=0$ at outflow, prescribed velocity at inflow and extrapolation on tranverse components.

We obtain $\mathbf{u}^{*}$ using the midpoint rule for temporal integration

$$
\mathbf{u}^{*}=\mathbf{u}^{n}+\Delta t\left(-[(\mathbf{u} \cdot \nabla) \mathbf{u}]^{n+\frac{1}{2}}-\frac{1}{\rho} \nabla p^{n-\frac{1}{2}}+\frac{\mu_{s}}{\rho} \Delta \mathbf{u}^{*}+\frac{1}{\rho}(\nabla \cdot \boldsymbol{\tau})^{n+\frac{1}{2}}\right)
$$

where the viscous stresses are calculated using the backward Euler method and the half time advective term is calculated using the edge velocities in (54). We note that $\mathbf{u}^{*}$ is not divergence-free by $O\left(\Delta t^{2}\right)$ because of a lagged pressure gradient.

The equation used to predict the solution to the viscous problem has the following form:

$$
\begin{aligned}
\mathbf{L}_{\nu} \mathbf{u}^{*}= & \mathbf{u}^{n}+\Delta t\left(-(\mathbf{u} \cdot \nabla) \mathbf{u}^{n+\frac{1}{2}}-\frac{1}{\rho} \nabla p^{n-\frac{1}{2}}+\frac{1}{\rho}\left(\mu_{s}+\frac{\left(\mu_{p}-\lambda a^{2}\right) \Delta t}{2 \lambda+\Delta t}\right) \Delta \mathbf{u}^{n}\right. \\
& \left.+\frac{1}{\rho} \frac{2 \lambda}{2 \lambda+\Delta t}(\nabla \cdot \tilde{\boldsymbol{\tau}})\right)
\end{aligned}
$$

where

$$
\mathbf{L}_{\nu}=\mathbf{I}-\frac{\Delta t}{\rho}\left(\mu_{s}+\frac{\left(\mu_{p}-\lambda a^{2}\right) \Delta t}{2 \lambda+\Delta t}\right) \Delta
$$

The boundary conditions for the viscous problem are $\mathbf{u} \cdot \hat{\mathbf{n}}=0$ at solid walls, $\frac{\partial \mathbf{u}}{\partial \hat{\mathbf{n}}}=0$ at outflow and prescribed velocity at inflow, which is implemented using residual correction.

A final projection operator is applied to the predicted velocity, $\mathbf{u}^{*}$, enforcing the incompressibility constraint on the velocity and updating the pressure gradient: 


$$
\begin{aligned}
\mathbf{u}^{n+1} & =\mathbf{u}^{*}-\nabla\left(\Delta^{-1}\left(\nabla \cdot \mathbf{u}^{*}\right)\right) \\
\nabla p^{n+\frac{1}{2}} & =\nabla p^{n-\frac{1}{2}}+\frac{\rho}{\Delta t} \nabla\left(\Delta^{-1}\left(\nabla \cdot \mathbf{u}^{*}\right)\right) .
\end{aligned}
$$

Projection boundary conditions are $\mathbf{u} \cdot \hat{\mathbf{n}}=0, \frac{\partial p}{\partial \hat{\mathbf{n}}}=0$ at solid walls, $\frac{\partial \mathbf{u}}{\partial \hat{\mathbf{n}}}=0$, $p=0$ at outflow and prescribed velocity with $\frac{\partial p}{\partial \hat{\mathbf{n}}}=0$ at inflow. Also, the final projection yields a divergence-free velocity field which contains unphysical checkerboard modes due to centered differencing. We use a simple, single-step filter to eliminate these modes $[15,20,8]$.

\subsection{Stress Discretization}

The stress discretization follows a similar algorithm as in [23]. First, we construct a Lax-Wendroff stress using only the elastic (hyperbolic) terms:

$$
\begin{aligned}
\tilde{\boldsymbol{\tau}}_{i+\frac{1}{2}, j, k}= & \frac{1}{2}\left(\boldsymbol{\tau}_{i, j, k}^{n}+\boldsymbol{\tau}_{i+1, j, k}^{n}\right)+ \\
& \frac{\Delta t}{2}\left(-(\mathbf{u} \cdot \nabla) \boldsymbol{\tau}+\nabla \mathbf{u} \cdot\left(\boldsymbol{\tau}+a^{2} \mathbf{I}\right)+\left(\boldsymbol{\tau}+a^{2} \mathbf{I}\right) \cdot \nabla \mathbf{u}^{T}\right)_{i+\frac{1}{2}, j, k}^{n} \cdot(60)
\end{aligned}
$$

We then predict a half time stress for the viscous problem, again, using the backward Euler method for viscous stresses

$$
\begin{aligned}
\boldsymbol{\tau}_{i+\frac{1}{2}, j, k}^{n+\frac{1}{2}} & =\tilde{\boldsymbol{\tau}}_{i+\frac{1}{2}, j, k}-\frac{\Delta t}{2}\left(\frac{1}{\lambda} \boldsymbol{\tau}_{i+\frac{1}{2}, j, k}^{n+\frac{1}{2}}-2\left(\frac{\mu_{p}}{\lambda}-a^{2}\right) \mathbf{D}\left(\mathbf{u}^{*}\right)\right) \\
& =\frac{2}{2 \lambda+\Delta t}\left(\lambda \tilde{\boldsymbol{\tau}}_{i+\frac{1}{2}, j, k}+\Delta t\left(\mu_{p}-\lambda a^{2}\right) \mathbf{D}\left(\mathbf{u}^{*}\right)\right)
\end{aligned}
$$

The stress is updated to the new time as follows:

$$
\begin{aligned}
\boldsymbol{\tau}^{n+1}= & \boldsymbol{\tau}^{n}+\Delta t\left(-(\mathbf{u} \cdot \nabla) \boldsymbol{\tau}+\nabla \mathbf{u} \cdot\left(\boldsymbol{\tau}+a^{2} \mathbf{I}\right)+\left(\boldsymbol{\tau}+a^{2} \mathbf{I}\right) \cdot \nabla \mathbf{u}^{T}\right)^{n+\frac{1}{2}} \\
& -\frac{\Delta t}{\lambda}\left(\boldsymbol{\tau}^{n+1}-2\left(\mu_{p}-a^{2} \lambda\right) \mathbf{D}\left(\mathbf{u}^{n+1}\right)\right) \\
= & \frac{\lambda}{\lambda+\Delta t}\left(\boldsymbol{\tau}^{n}+\Delta t\left[\left\{-[(\mathbf{u} \cdot \nabla) \boldsymbol{\tau}]+\left(\nabla \mathbf{u} \cdot\left(\boldsymbol{\tau}+a^{2} \mathbf{I}\right)\right)+\right.\right.\right. \\
& \left.\left.\left.\left(\left(\boldsymbol{\tau}+a^{2} \mathbf{I}\right) \cdot \nabla \mathbf{u}^{T}\right)\right\}^{n+\frac{1}{2}}+2\left(\frac{\mu_{p}}{\lambda}-a^{2}\right) \mathbf{D}\left(\mathbf{u}^{n+1}\right)\right]\right) .
\end{aligned}
$$

Stress boundary conditions are implemented numerically using a zeroth-order extrapolation. For the normal components of the stress this condition is justified by the characteristic analysis in section 2.1 plus the assumption that 
a uniform elastic stress distribution will, by itself, generate no waves. The tangential components on boundary edges are not used.

\subsection{Stability}

We have applied von Neumann stability analysis to the Lax-Wendroff method in general. In $2 \mathrm{D}$ we consider the scalar advection equation

$$
\frac{\partial u}{\partial t}+a \frac{\partial u}{\partial x}+b \frac{\partial u}{\partial y}=0
$$

on a periodic domain with given initial conditions [9]. A sufficient condition for the method to be stable in the long wavelength limit is

$$
\sigma_{x}^{2} n_{x}^{4}+\sigma_{y}^{2} n_{x}^{4}>\left(\sigma_{x} n_{x}+\sigma_{y} n_{x}\right)^{4}
$$

where $\sigma_{x}=\frac{a \Delta t}{\Delta x}, \sigma_{y}=\frac{b \Delta t}{\Delta y}$ and $\left(n_{x}, n_{y}\right)$ are components of the unit normal for the orthonormal basis formed by the complex exponentials on the given interval. This sufficiency condition yields a CFL number of $\left|\sigma_{x}\right|,\left|\sigma_{y}\right|<\frac{1}{4}$. In $3 \mathrm{D}$, we obtain a slightly more restrictive CFL of $\left|\sigma_{x}\right|,\left|\sigma_{y}\right|,\left|\sigma_{z}\right|<\frac{1}{9}$.

For our method a stable time step is obtained from the following advective CFL condition $\left(\sigma<\frac{1}{9}\right)$ :

$$
\begin{gathered}
\max _{i, j, k}\left[\left|u_{i, j, k}\right|+\left(2\left(\tau_{x x}+a^{2}\right) / \rho\right)^{\frac{1}{2}}\right] \Delta t<\sigma \Delta x \\
\max _{i, j, k}\left[\left|v_{i, j, k}\right|+\left(2\left(\tau_{y y}+a^{2}\right) / \rho\right)^{\frac{1}{2}}\right] \Delta t<\sigma \Delta y \\
\max _{i, j, k}\left[\left|w_{i, j, k}\right|+\left(2\left(\tau_{z z}+a^{2}\right) / \rho\right)^{\frac{1}{2}}\right] \Delta t<\sigma \Delta z .
\end{gathered}
$$

\subsection{Viscoelastic Limits of Algorithm}

The viscous limit of our method, $\lambda=0 \Longrightarrow a^{2}=0$, reveals the stress of a Newtonian fluid with augmented viscosity due to the polymer:

$$
\begin{aligned}
\boldsymbol{\tau}_{i+\frac{1}{2}, j, k}^{n+\frac{1}{2}} & =2 \mu_{p} \mathbf{D}\left(\mathbf{u}^{*}\right) \\
\boldsymbol{\tau}^{n+1} & =2 \mu_{p} \mathbf{D}\left(\mathbf{u}^{n+1}\right)
\end{aligned}
$$




$$
\mathbf{L}_{\nu}=\mathbf{I}-\frac{\left(\mu_{s}+\mu_{p}\right) \Delta t}{\rho} \Delta .
$$

The elastic limit of the method, $\lambda \rightarrow \infty, \mu_{p} / \lambda$ finite $\Longrightarrow a^{2} \rightarrow \mu_{p} / \lambda$, demonstrates the hyperbolic nature, with finite, non-zero wavespeed, which the stress discretization assumes in this limit, with no viscous contribution by the polymer:

$$
\begin{aligned}
\boldsymbol{\tau}_{i+\frac{1}{2}, j, k}^{n+\frac{1}{2}}= & \tilde{\boldsymbol{\tau}}_{i+\frac{1}{2}, j, k} \\
= & \frac{1}{2}\left(\boldsymbol{\tau}_{i, j, k}^{n}+\boldsymbol{\tau}_{i+1, j, k}^{n}\right) \\
& +\frac{\Delta t}{2}\left(-(\mathbf{u} \cdot \nabla) \boldsymbol{\tau}+\nabla \mathbf{u} \cdot\left(\boldsymbol{\tau}+\frac{\mu_{p}}{\lambda} \mathbf{I}\right)+\left(\boldsymbol{\tau}+\frac{\mu_{p}}{\lambda} \mathbf{I}\right) \cdot \nabla \mathbf{u}^{T}\right)_{i+\frac{1}{2}, j, k}^{n}(74) \\
\boldsymbol{\tau}^{n+1}= & \boldsymbol{\tau}^{n}+\Delta t\left(-(\mathbf{u} \cdot \nabla) \boldsymbol{\tau}+\nabla \mathbf{u} \cdot\left(\boldsymbol{\tau}+\frac{\mu_{p}}{\lambda} \mathbf{I}\right)+\left(\boldsymbol{\tau}+\frac{\mu_{p}}{\lambda} \mathbf{I}\right) \cdot \nabla \mathbf{u}^{T}\right)^{n+\frac{1}{2}}(75) \\
\mathbf{L}_{\nu}= & \mathbf{I}-\frac{\mu_{s} \Delta t}{\rho} \Delta .
\end{aligned}
$$

For Maxwell fluids where $\mu_{s}=0$ we note that no-slip boundary conditions are not imposed because the viscous terms vanish.

\section{Results}

We apply the method to unsteady flow of Oldroyd-B and Maxwell fluids in abrupt planar contraction channels (Figure 1). First, we simulate the OldroydB fluid without viscosity $\left(\mu_{s}=0\right)$ in sudden contraction channels to demonstrate stable and convergent calculation of the flow of an unsteady Maxwell fluid, which allows finite elastic wave propagation. We then add viscosity to the Maxwell fluid (Oldroyd-B) and demonstrate stable and convergent simulations of high $W e$ in abrupt contractions for unsteady flow with hybrid viscous and elastic behavior. The method is valid for both 2D and 3D flows, though only $2 \mathrm{D}$ results are shown here for simplicity in demonstrating the robustness of the algorithm. For 3D results we refer to our previous work in [23]. We also present results for the viscous limit of an Oldroyd-B fluid in abrupt contraction channels. We characterize our results with the elastic Mach number and Weissenberg number where the characteristic velocity, $U$, is the average velocity in the contraction channel, or the average inflow velocity times the contraction ratio. We demonstrate accuracy of the method for each case. For viscous flows convergence results are obtained away from the inlet of the channel in order to isolate the re-entrant corner and to omit known singularities at the inlet due to prescribed constant velocity boundary conditions. 


\subsection{Flow of a Maxwell Fluid}

The example which best demonstrates the robustness of our method is the unsteady flow of a Maxwell fluid. Again, the Maxwell fluid is the Oldroyd-B fluid with $\mu_{s}=0$; also, propagation of waves with finite elastic wave speeds is allowed. We show results for flow of a Maxwell fluid in both 2:1 and 4:1 contractions. Figures 2 and 3 are time sequences of velocity that demonstrate convergence for $W e=205.6, M a=.31$ in a $2: 1$ contraction and $W e=822.4$, $M a=.62$ in a $4: 1$ contraction, respectively. Figures 4 and 5 show the difference between pressures for the viscous and elastic limits, clearly demonstrating the wave behavior in the Maxwell fluid. In Tables I and II we show convergence results for the Maxwell fluid at very long time: $M a=0.3, W e=205.6$ in 2:1 contraction; $M a=0.6, W e=822.4$ in $4: 1$ contraction. The solution shows second-order convergence in $L_{1}$ and $L_{2}$ norms and only first-order convergence in $L_{\infty}$ norm. We attribute the slight drop off in convergence in the $L_{\infty}$ norm to the geometric singularity and wave interactions. We note again that the no-slip boundary condition is not imposed because of the vanishing viscous terms in these flows.

We make several observations from the flow data in the Maxwell fluid case. First, we note that elastic wave behavior is captured in these results using the same algorithm and same stability condition as the results generated for the more viscous fluids seen in later sections. Second, these results are valid for very long time without violating hyperbolicity and while maintaining stability and convergence, even though there is a build up of stress at corners. Third, results for the Maxwell fluid are not limited by $W e$, but only subject to subcritical elastic Mach number which requires $\mu_{p} / \lambda$ to be finite for wellposedness in the elastic limit, or $\mu_{p} / \lambda>\rho U^{2}$. Finally, we have not physically validated the computed behavior, though the solution does clearly converge and is not a numerical artifact.

\subsection{Elastic Flow of an Oldroyd-B Fluid}

We have also applied the method to elastic flows of Oldroyd-B fluids in planar contractions to demonstrate mixed viscous and elastic effects. For regimes where the solvent viscosity is large enough we note not much difference in the solution from viscous limit examples. However, for hybrid flows, where the elastic waves interact with viscous forces we find that there is definite unsteady behavior. Time sequences of velocity in 2:1 $(M a=0.06, W e=8)$ and 4:1 contractions $(M a=0.12, W e=32)$ are shown in Figures 6 and 7, respectively, demonstrating that the flow is viscous, though not a classic parabolic velocity profile due to the effect of elastic shear waves. More experimentation is needed 
to quantify the extent of this behavior and to determine the critical values of $W e$ at which the transition to hybrid, unsteady behavior occurs [16]. In Tables III and IV we show convergence rates for the Oldroyd-B fluid at early times. In Tables V, VI and VII we show convergence rates for the Oldroyd-B fluid at late times. The solution does not show second-order convergence, as expected, due to the use of backward Euler for viscous stresses, but it is better than first-order because the flow is not purely viscous. We attribute the drop off in convergence in the $L_{\infty}$ norm to the presence of the viscous effects interacting with elastic waves near the corner singularity. We also note the drop off in the $L_{\infty}$ norm at the late times, seen in Table VII and the last frames of Figure 7 for the $4: 1$ contraction.

\subsection{Viscous Limit of Oldroyd-B}

We demonstrate convergence of velocity in the viscous limit $(W e=0)$ of an Oldroyd-B fluid for both 2:1 and 4:1 contractions in Figure 8. We note singularities at both inflow (due to constant velocity boundary condition) and re-entrant corners. We show convergence rates for this data in Tables VIII and IX. The convergence rates are first-order, as expected, due to the use of backward Euler for viscous stresses. In the $L_{\infty}$ norm the results are $O\left(h^{\frac{1}{2}}\right)$ due to the presence of geometric singularities at re-entrant corners. We also note the restriction on the time step in the viscous limit. The maximum advective velocity for these flows is approximately 0.05 while the wavespeed which uses $a^{2}$ is about 1.5, demonstrating the slight restriction on time step in our method, about a factor of 30. However, if the elastic wavespeed is used to determine the time step in this viscous case as in [14], then the restriction is much greater, or a factor of $10^{6}$.

\section{Conclusions}

We demonstrate a stable and convergent numerical method for viscoelastic flow in abrupt contraction channels. The method is different from prior steadystate or implicit methods in that it is an appropriately designed unsteady flow method which can capture wave motion in strongly elastic flows for any value of the Weissenberg number and is only restricted by subcritical elastic Mach number. The method requires a single CFL condition for the range of elastic flows, from the viscous limit to the elastic limit. This is achieved through a variation of the BCG method which obeys the desired viscous and elastic limits of the equations of motion. For Maxwell fluids we demonstrate unsteady results for arbitrarily high $W e$ and $M a<1$ where elastic shear waves propagate in an incompressible fluid and are captured by the method 
in a stable and convergent manner. The method has also been shown to be stable and convergent for unsteady high Weissenberg number flows of OldroydB fluids in abrupt contractions. More experimentation is needed to quantify the extent of the behavior (vortex enhancement, for example) in these flows. The method also calculates the viscous limit of the Oldroyd-B equation, with a time step restricted only slightly more than that governed by the advective velocity alone. The method compares very well to known exact solutions and preliminary data from experimental flows in microfluidic devices [23].

In the future we will modify the scheme in several ways. To improve the accuracy of viscous flows to second-order, we will implement the implicit RungeKutta scheme of Twizell, Gumel and Arigu in [24] for the viscous stresses as it is known to be stable in the presence of boundaries, unlike Crank-Nicolson [10]. We will also take advantage of the ability to cast the PDEs (5) and (7) in a hybrid conservation form which exploits hyperbolicity while maintaining the viscous and elastic limits. This means we will naturally extend the method to make use of the higher-order Godunov method of the BCG formulation, allowing us to eliminate the current subcritical Mach number restriction. Higherorder Godunov methods are more robust than LW for hyperbolic systems; and the CFL time step is an order of magnitude larger than that of the LW method used here. Therefore, for certain applications such as particle-laden flow in microfluidic devices and other complex microscale geometries where sharp gradients (or even waves) exist it would be advantageous to develop BCG for viscoelasticity. Finally, though biological flows in the planar geometries of microfluidic devices are the application of interest, a more robust test of the algorithm would be to simulate vortex enhancement seen in experimental flows through axisymmetric contractions. In this we would also like to extend the scheme to other viscoelastic constitutive equations because the Oldroyd-B model does not provide a realistic description of polymeric fluids, though it serves well as a test bed for the numerical method. FENE-type equations, for example, are more physical as they model nonlinear elastic effects in the fluid.

\section{Acknowledgements}

This work was performed under the auspices of the U.S. Department of Energy by the University of California, Lawrence Livermore National Laboratory under contract No. W-7405-Eng-48. Work at the Lawrence Berkeley National Laboratory was supported by the US DOE Mathematical, Information, and Computer Sciences (MICS) Division under contract number DE-AC0376SF00098. Work at the University of California, Davis was partially support by the US DOE MICS Division under contract number DE-FG02-03ER25579. 


\section{References}

[1] M. Aboubacar, H. Matallah, and M. F. Webster. Higly elastic solutions for Oldroyd-B and Phan-Thien/Tanner fluids with a finite volume/element method: planar contraction flows. J. Non-Newtonian Fluid Mech., 103:65-103, 2002.

[2] A. Almgren, J. B. Bell, P. Colella, and L. H. Howell. A conservative adaptive projection method for the variable density incompressible NavierStokes equations. J. Comput. Phys., 142(1):1-46, 1998.

[3] J. B. Bell, P. Colella, and H. M. Glaz. A second-order projection method for the incompressible Navier-Stokes equations. J. Comput. Phys., 85:257-283, 1989.

[4] R. B. Bird, R. C. Armstrong, and O. Hassager. Dynamics of Polymeric Liquids. Wiley, New York, 1977.

[5] A. J. Chorin. Numerical solutions of the Navier-Stokes equations. Math. Comp., 22:745-762, 1968.

[6] M. J. Crochet, A. R. Davies, and K. Walters. Numerical Simulation of NonNewtonian Flow. Elsevier, Amsterdam, 1984.

[7] M. J. Crochet and G. Pilate. Plane flow of a fluid of second grade through a contraction. J. Non-Newtonian Fluid Mech., 1:247-258, 1976.

[8] R. Crockett, P. Colella, R. Fisher, R. I. Klein, and C. F. McKee. An unsplit, cell-centered Godunov method for ideal MHD. to appear.

[9] J. Hoffman. Numerical Methods for Engineers and Scientists. McGraw-Hill, 1992.

[10] H. Johansen and P. Colella. A Cartesian grid embedded boundary method for Poisson's equation on irregular domains. J. Comput. Phys., 147:60-85, 1998.

[11] D. D. Joseph. Fluid Dynamics of Viscoelastic Liquids. Springer-Verlag, New York, 1990.

[12] D. D. Joseph, M. Renardy, and J.-C. Saut. Hyperbolicity and change of type in the flow of viscoelastic fluids. Arch. Ration. Mech. Anal., 87:213-251, 1985.

[13] R. Keunings. On the high Weissenberg number problem. J. Non-Newtonian Fluid Mech., 20:209-226, 1986.

[14] R. Kupferman. Simulation of viscoelastic fluids: Couette-Taylor flow. $J$. Comput. Phys., 147:22-59, 1998.

[15] M. Lai. A projection method for reacting flow in the zero Mach number limit. PhD thesis, University of California, Berkeley, 1994.

[16] J. V. Lawler, S. J. Muller, R. A. Brown, and R. C. Armstrong. Laser Doppler velocimetry measurements of velocity fields and transitions in viscoelastic fluids. J. Non-Newtonian Fluid Mech., 20:51-92, 1986. 
[17] G. G. Lipscomb, R. Keunings, and M. M. Denn. Implications of boundary singularities in complex geometries. J. Non-Newtonian Fluid Mech., 24:85-96, 1987.

[18] J. M. Marchal and M. J. Crochet. A new mixed finite element for calculating viscoelastic flow. J. Non-Newtonian Fluid Mech., 26:77-114, 1987.

[19] T. N. Phillips and A. J. Williams. Viscoelastic flow through a planar contraction using a semi-Lagrangian finite volume method. J. Non-Newtonian Fluid Mech., $87: 215-246,1999$.

[20] W. J. Rider. Filtering nonsolenoidal modes in numerical solutions of incompressible flows. Technical Report LA-UR-3014, Los Alamos National Laboratory, 1994.

[21] I. M. Rutkevich. The propagation of small perturbations in a viscoelastic fluid. Appl. Math. Mech., 34:35-50, 1970.

[22] I. M. Rutkevich. On the thermodynamic interpretation of the evolutionary conditions of the equations of the mechanics of finitely deformableviscoelastic media of maxwell type. Appl. Math. Mech., 36:283-295, 1972.

[23] D. Trebotich, P. Colella, G. Miller, and D. Liepmann. A numerical model of viscoelastic flow in microchannels. In Technical Proceedings of the Sixth International Conference on Modeling and Simulation of Microsystems, San Francisco, CA, Feb 23-27 2003. Applied Computational Research Society, Cambridge, MA.

[24] E. H. Twizell, A. B. Gumel, and M. A. Arigu. Second-order $L_{0}$-stable methods for the heat equation with time-dependent boundary conditions. Adv. Comp. Math., 6(3):333-352, 1996.

[25] J. S. Ultman and M. M. Denn. Anomolous heat transfer and a wave phenomenon in dilute polymer solutions. Trans. Soc. Rheology, 14:307-317, 1970.

[26] S.-C. Xue, N. Phan-Thien, and R. I. Tanner. Three dimensional numerical simulations of viscoelastic flows through planar contractions. J. Non-Newtonian Fluid Mech., 74:195-245, 1998. 
List of Figures

Figure 1. 2D illustration of viscous flow in a planar contraction channel with axial direction $z$ and transverse direction $x$.

Figure 2. Convergence of Maxwell fluid in 2:1 contraction, $M a=0.3, W e=$ 205.6. (L) 200 time step increments, $h=1 / 128$; (R) 400 time step increments, $h=1 / 256$.

Figure 3. Convergence of Maxwell fluid in 4:1 contraction, $M a=0.6, W e=$ 822.4. (L) 200 time step increments, $h=1 / 128$; (R) 400 time step increments, $h=1 / 256$.

Figure 4. Pressure in elastic, viscous limits for 2:1 contraction. Normalized to $p_{\text {max }}^{\text {elastic }}=0.03$ bar; $p_{\text {max }}^{\text {viscous }}=16$ bar.

Figure 5. Pressure in elastic, viscous limits for 4:1 contraction. Normalized to $p_{\max }^{\text {elastic }}=0.07$ bar; $p_{\max }^{\text {viscous }}=116$ bar.

Figure 6. Convergence of Oldroyd-B fluid in 2:1 contraction, $M a=0.06$, $W e=8$. (L) 200 time step increments, $h=1 / 128 ;(\mathrm{R}) 400$ time step increments, $h=1 / 256$.

Figure 7. Convergence of Oldroyd-B fluid in 4:1 contraction, $M a=0.13$, $W e=32$. (L) 200 time step increments, $h=1 / 128$; (R) 400 time step increments, $h=1 / 256$.

Figure 8. Convergence of viscous limit $(W e=0)$ of Oldroyd-B fluid at 2000 time steps. (L) $h=1 / 128 ;(\mathrm{R}) h=1 / 256$. 


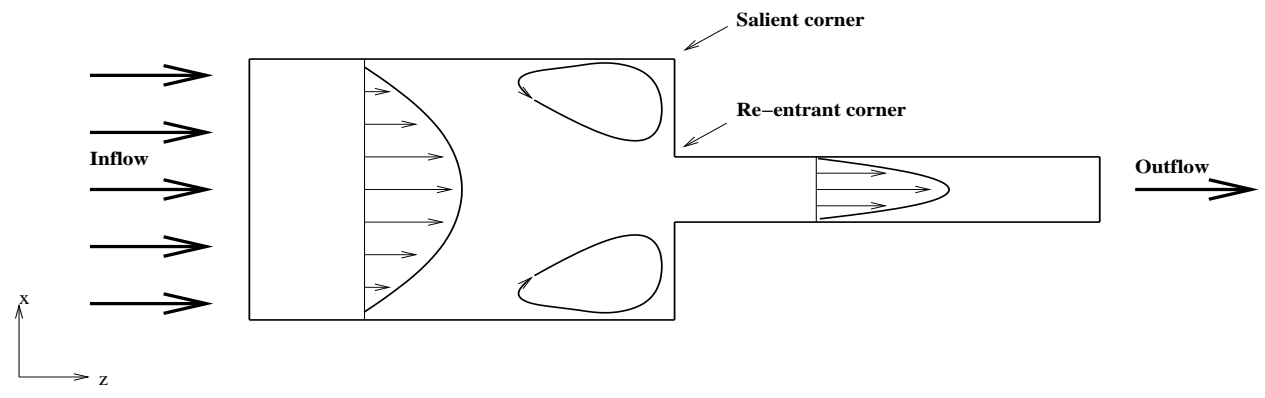

Fig. 1. 2D illustration of viscous flow in a planar contraction channel with axial direction $z$ and transverse direction $x$. 

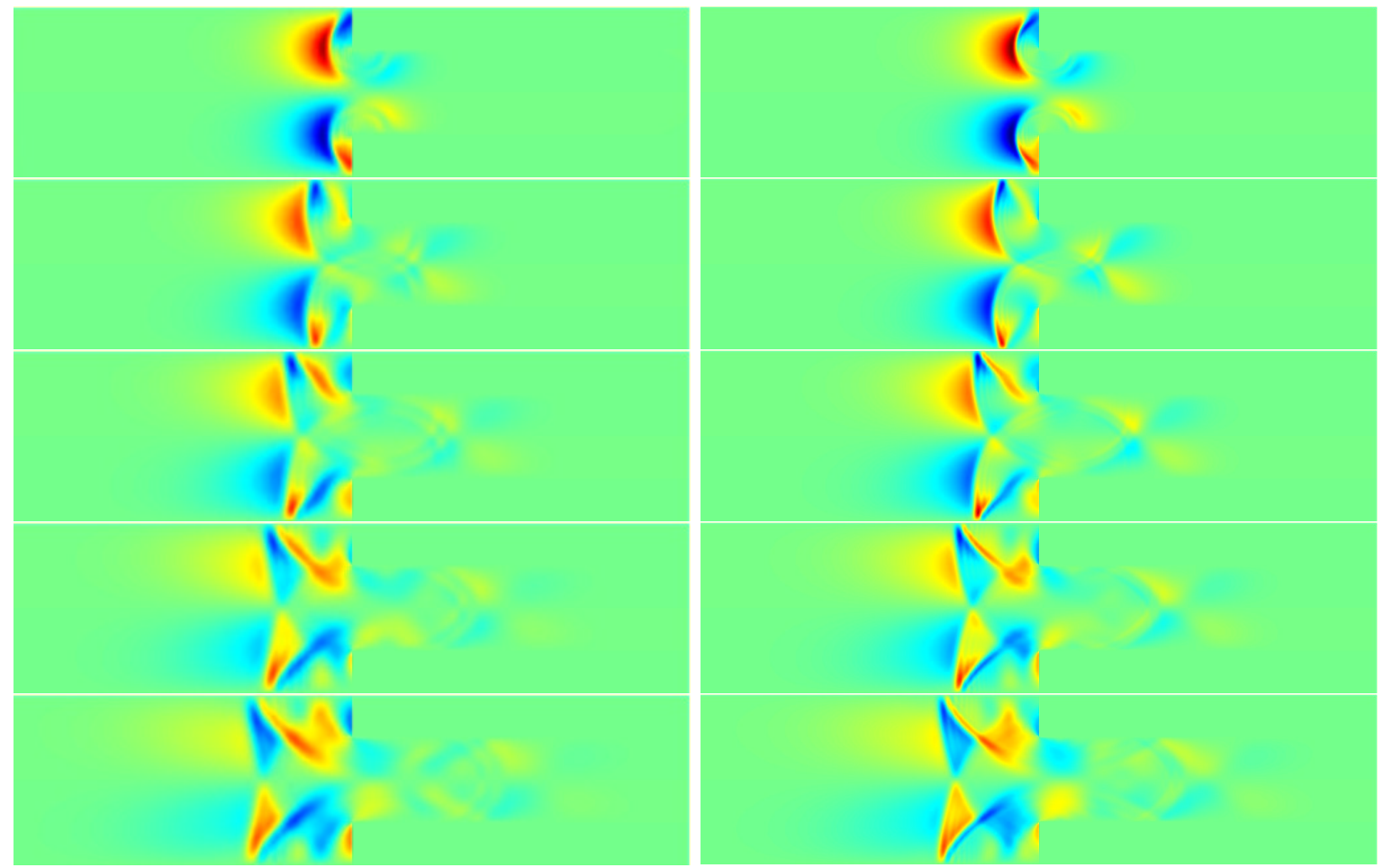

(a) Transverse velocity, $u,-0.021$ (blue) to 0.021 (red) $\mathrm{cm} / \mathrm{sec}$.
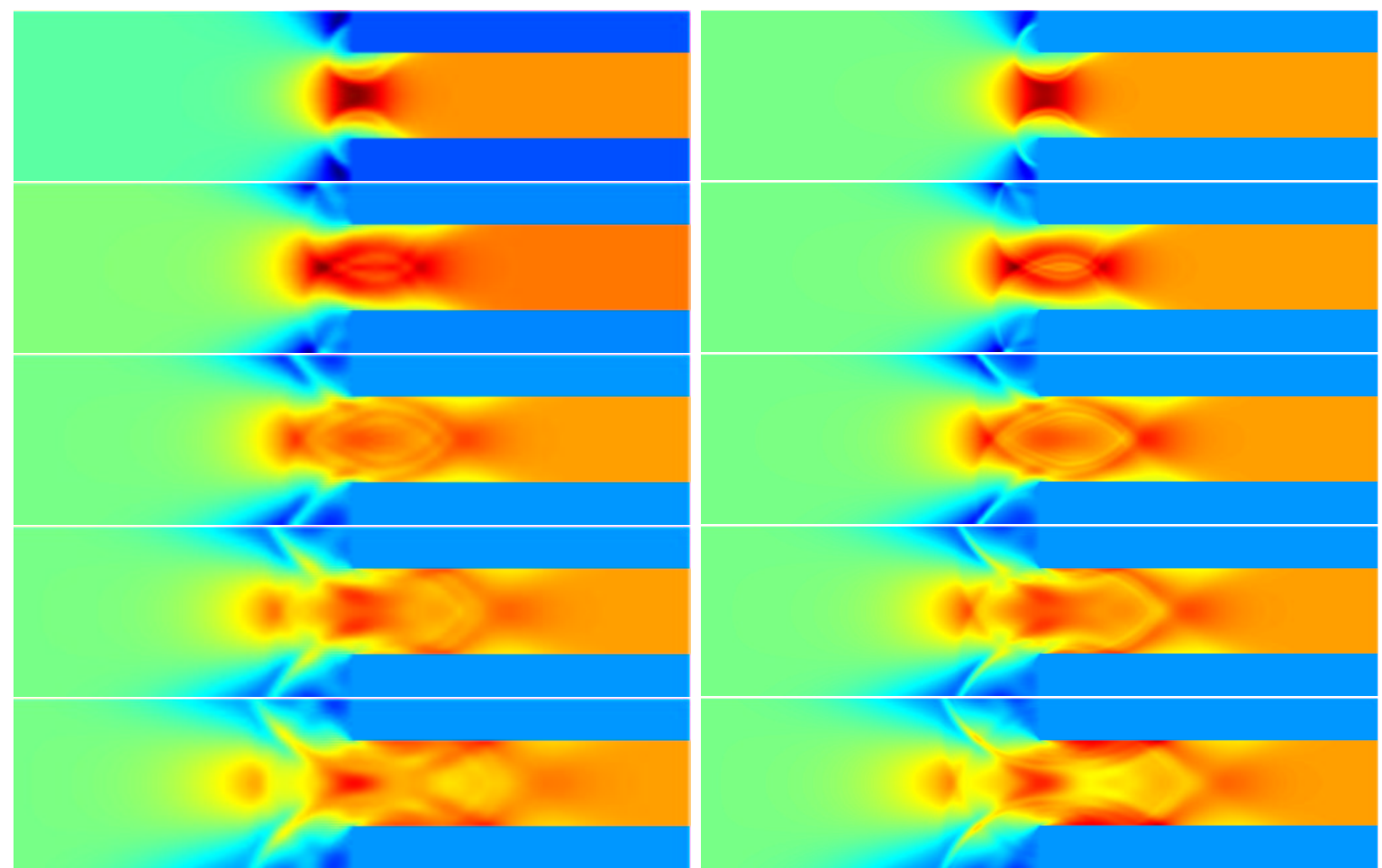

(b) Axial velocity, w, -1.2 (blue) to 3.2 (red) cm/sec.

Fig. 2. Convergence of Maxwell fluid in 2:1 contraction, $M a=0.3, W e=205.6$.

(L) 200 time step increments, $h=1 / 128$; (R) 400 time step increments, $h=1 / 256$. 

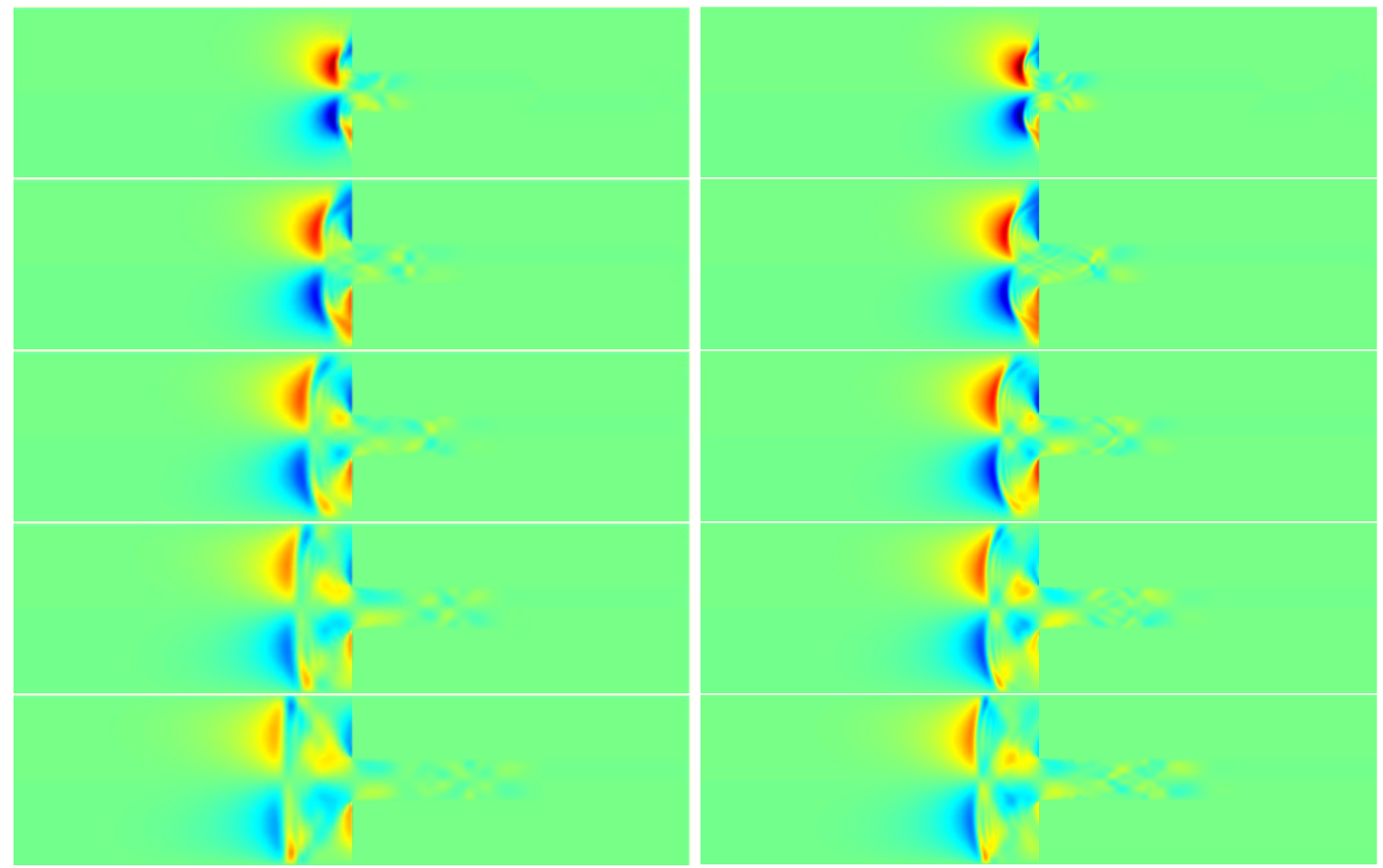

(a) Transverse velocity, $u,-0.04$ (blue) to 0.04 (red) $\mathrm{cm} / \mathrm{sec}$.
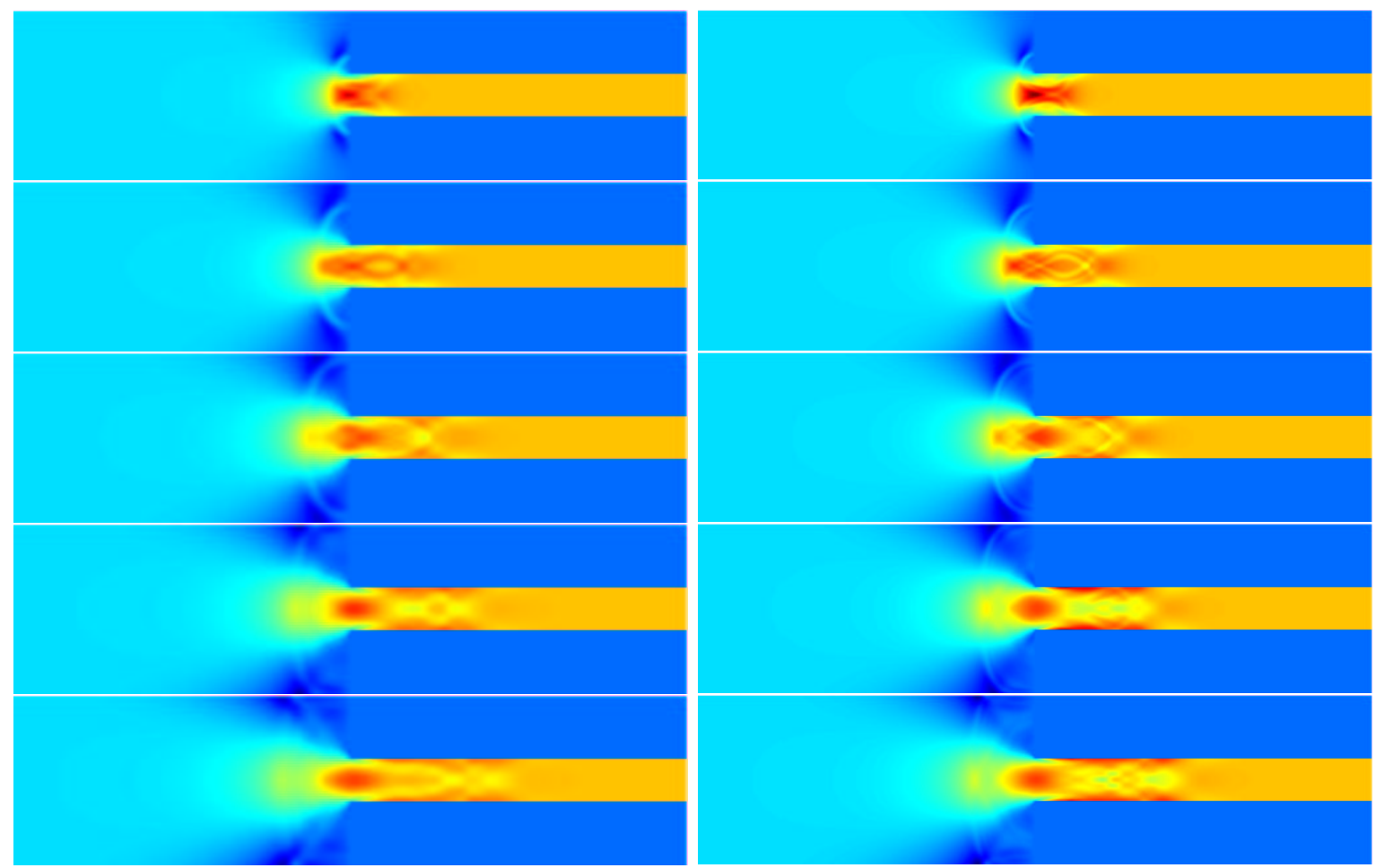

(b) Axial velocity, w, -2.0 (blue) to 6.6 (red) $\mathrm{cm} / \mathrm{sec}$.

Fig. 3. Convergence of Maxwell fluid in 4:1 contraction, $M a=0.6$, $W e=822.4$.

(L) 200 time step increments, $h=1 / 128$; (R) 400 time step increments, $h=1 / 256$. 


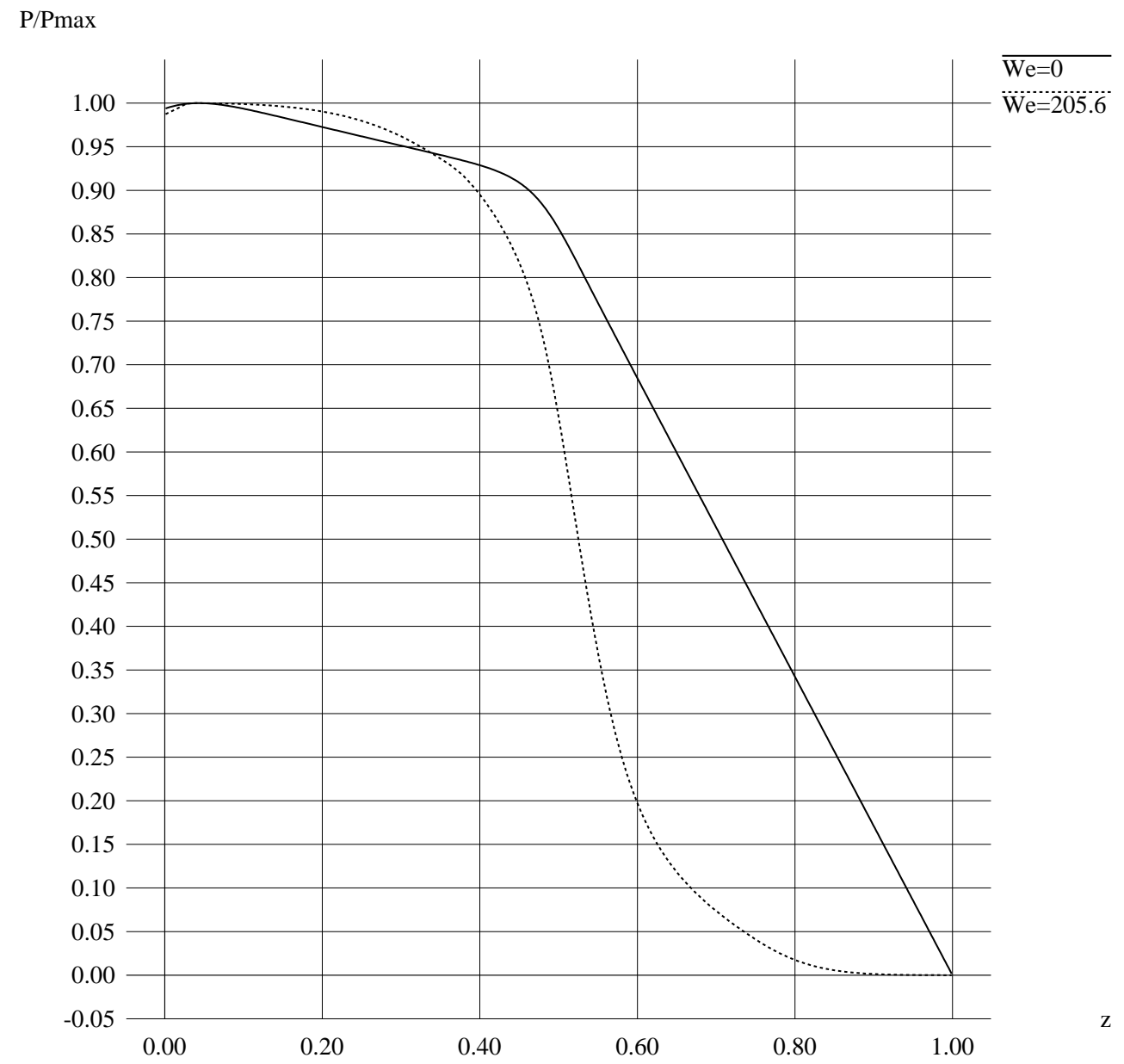

Fig. 4. Pressure in elastic, viscous limits for 2:1 contraction. Normalized to $p_{\max }^{\text {elastic }}=0.03 \mathrm{bar} ; p_{\max }^{\text {viscous }}=16 \mathrm{bar}$. 


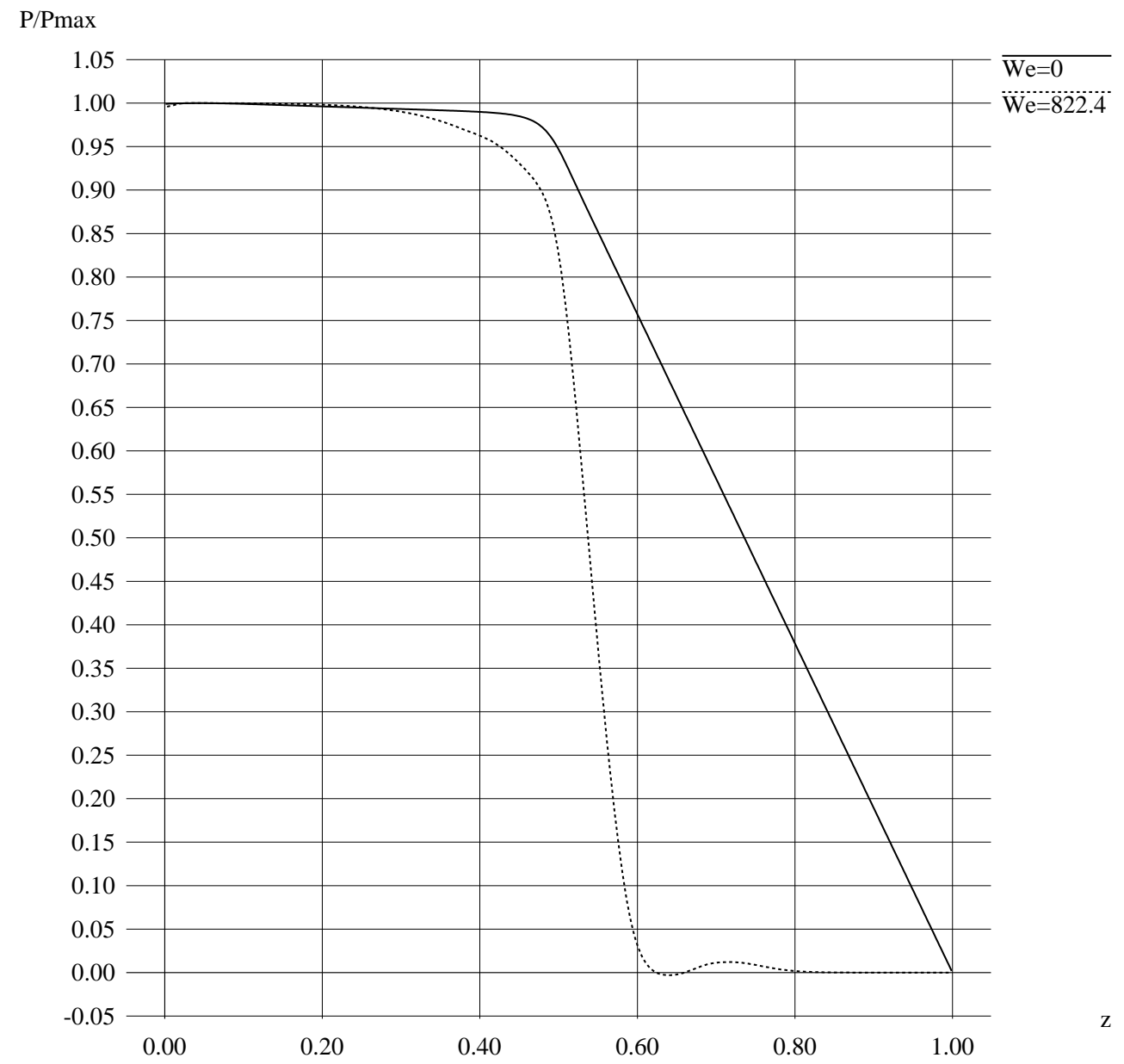

Fig. 5. Pressure in elastic, viscous limits for $4: 1$ contraction. Normalized to $p_{\max }^{\text {elastic }}=0.07$ bar; $p_{\max }^{\text {viscous }}=116$ bar. 

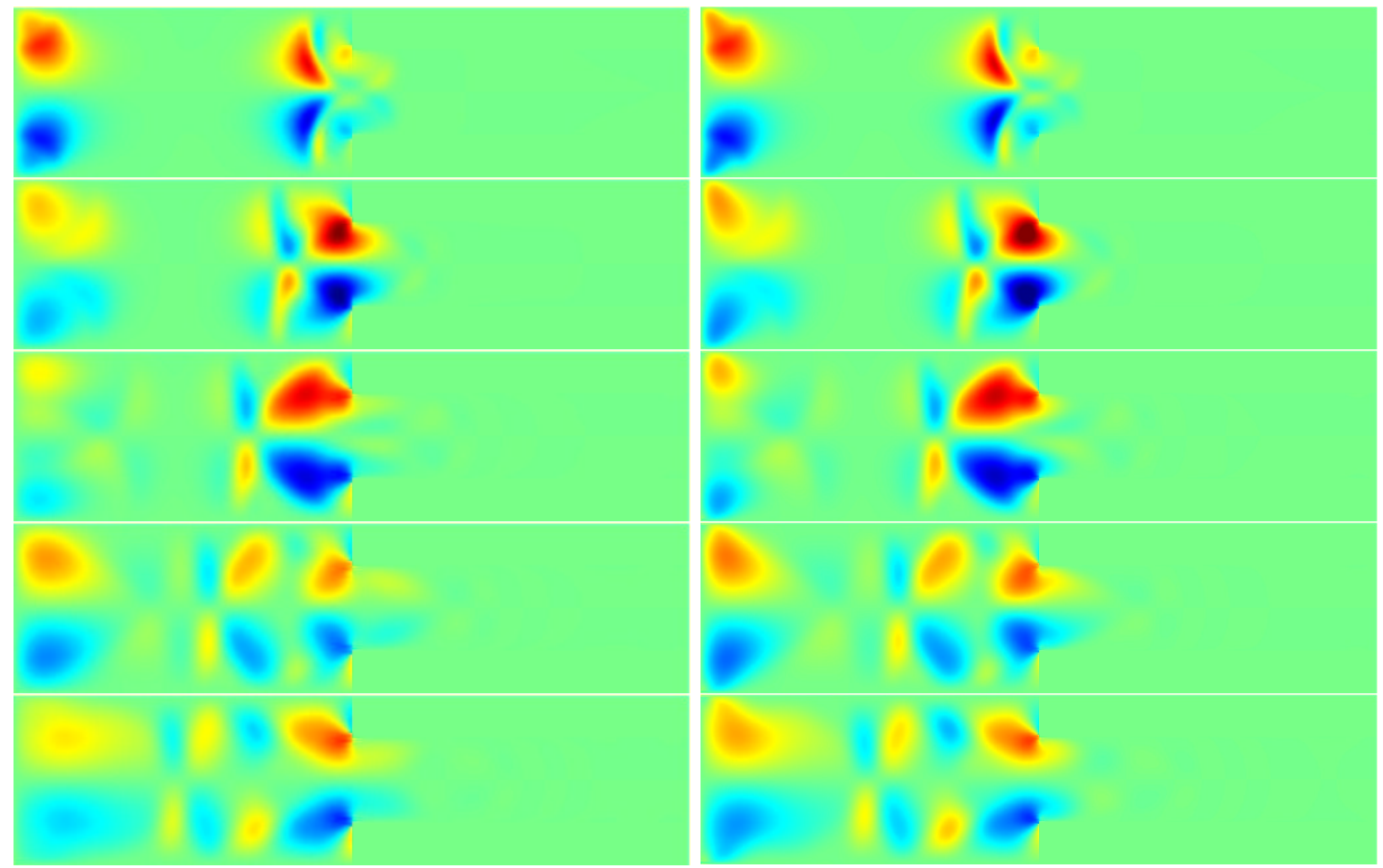

(a) Transverse velocity, $u,-0.07$ (blue) to 0.07 (red) $\mathrm{cm} / \mathrm{sec}$.
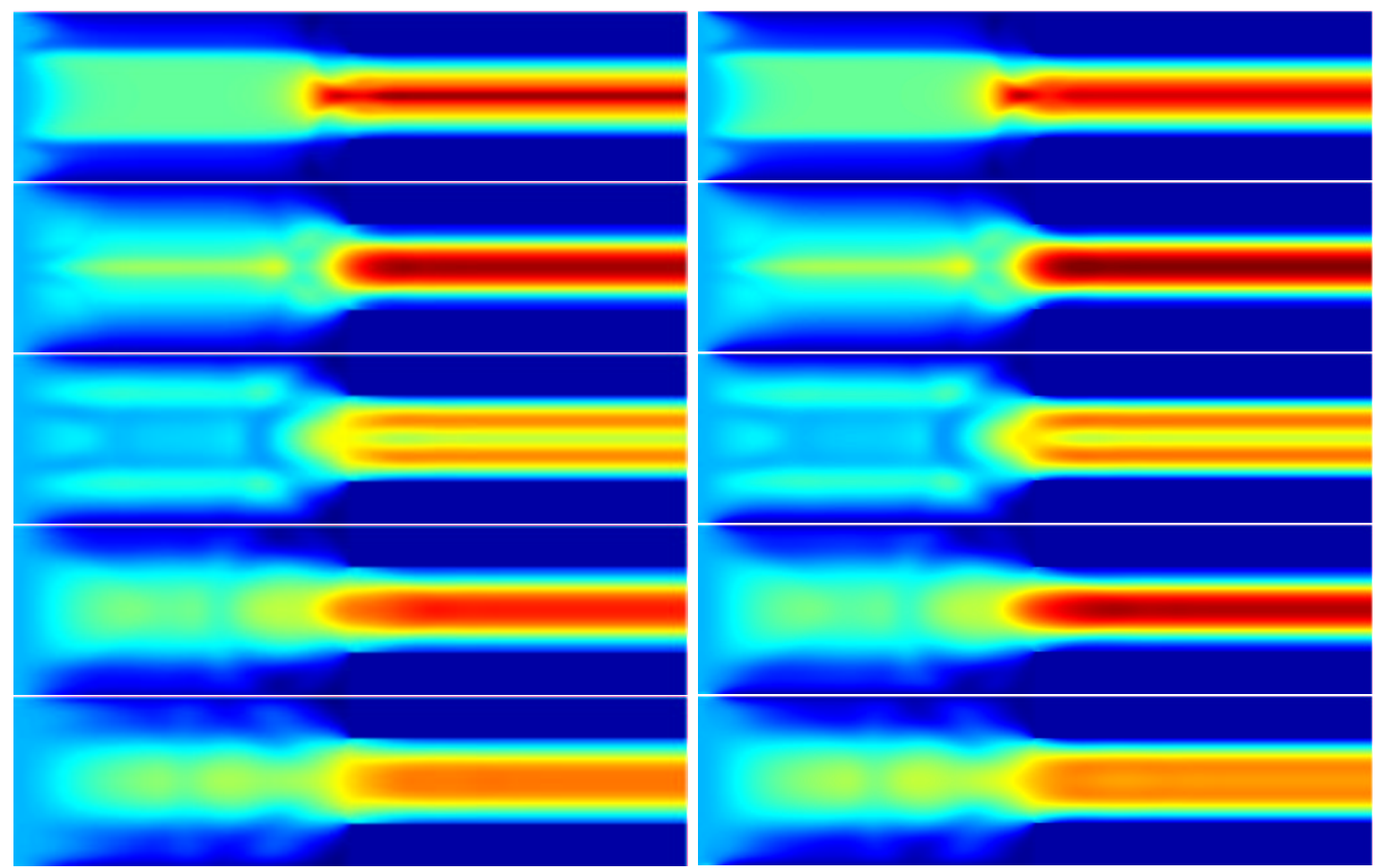

(b) Axial velocity, $w,-0.1$ (blue) to 3.5 (red) cm/sec.

Fig. 6. Convergence of Oldroyd-B fluid in 2:1 contraction, $M a=0.06, W e=8$.

(L) 200 time step increments, $h=1 / 128$; (R) 400 time step increments, $h=1 / 256$. 

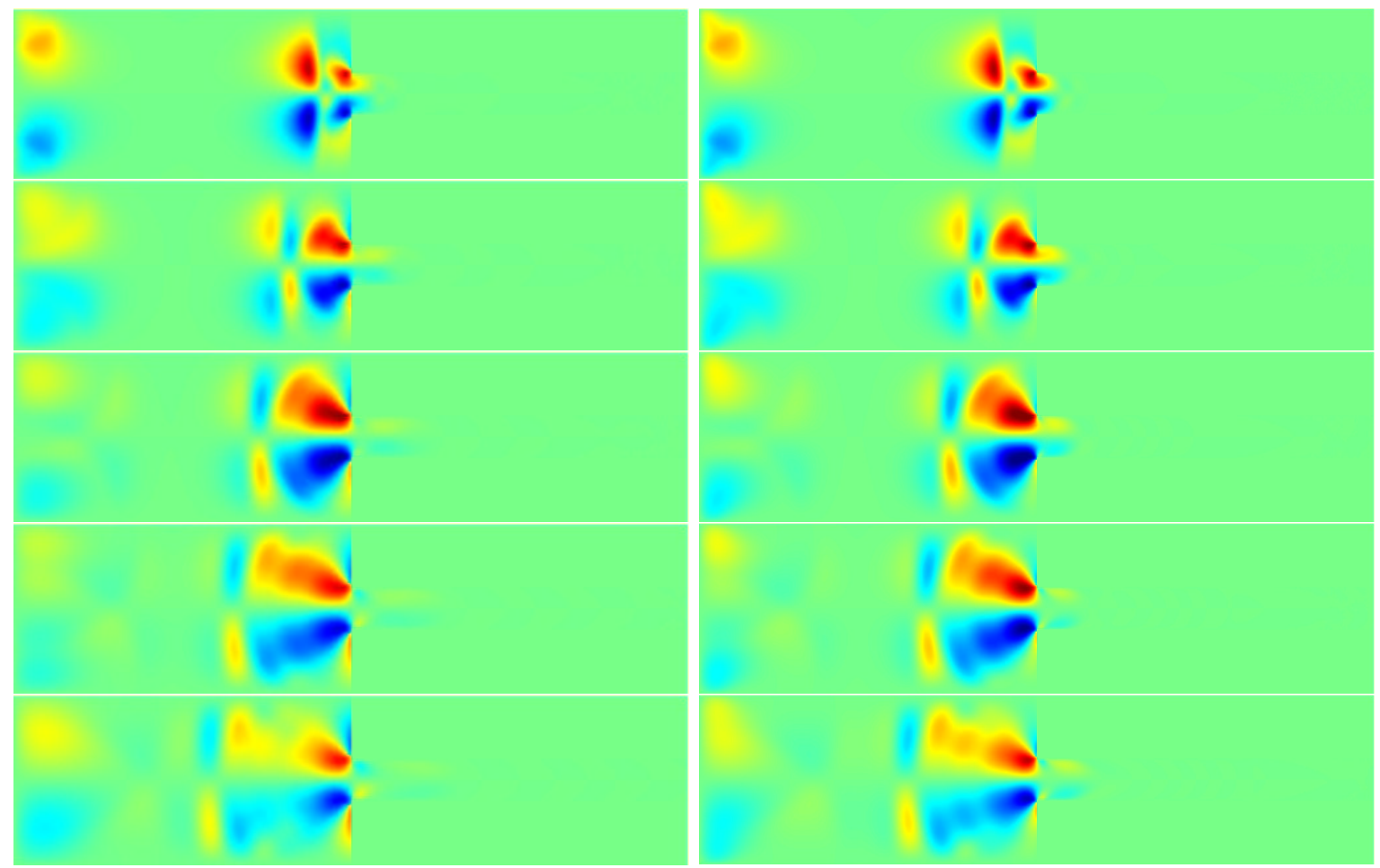

(a) Transverse velocity, $u,-0.12$ (blue) to 0.12 (red) $\mathrm{cm} / \mathrm{sec}$.
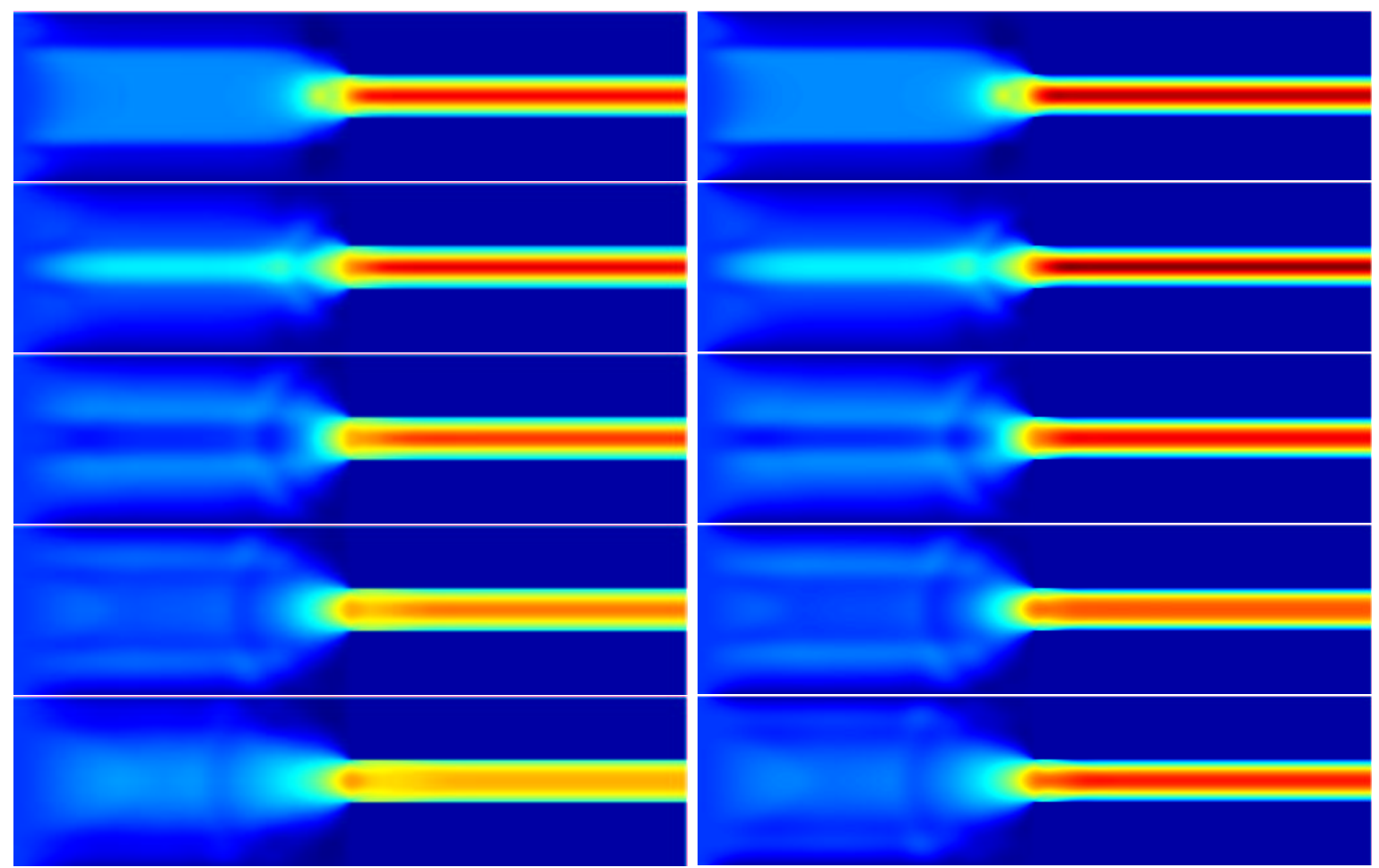

(b) Axial velocity, $w,-0.2$ (blue) to 6.5 (red) $\mathrm{cm} / \mathrm{sec}$.

Fig. 7. Convergence of Oldroyd-B fluid in 4:1 contraction, $M a=0.13, W e=32$.

(L) 200 time step increments, $h=1 / 128$; (R) 400 time step increments, $h=1 / 256$. 

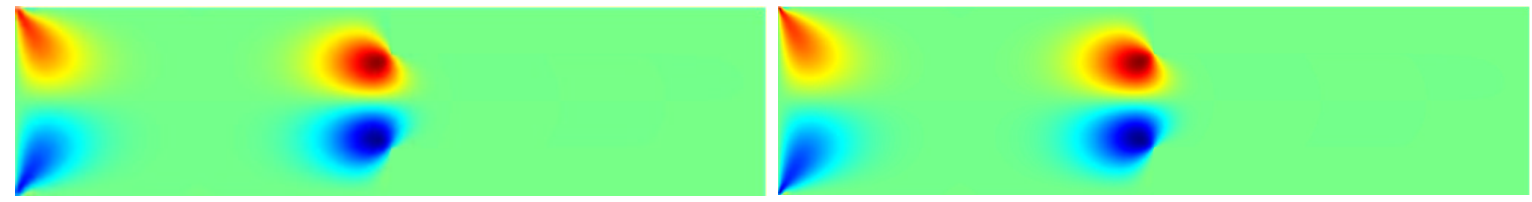

(a) Transverse velocity, $u,-0.009$ (blue) to 0.009 (red) $\mathrm{cm} / \mathrm{sec}, 2: 1$.
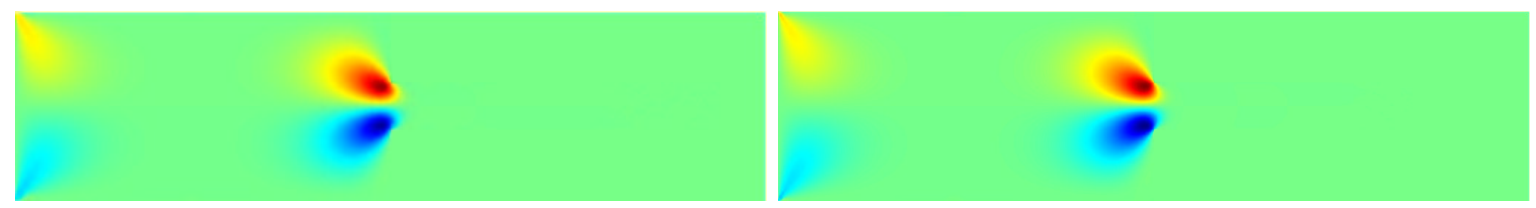

(b) Transverse velocity, $u,-0.02$ (blue) to 0.02 (red) $\mathrm{cm} / \mathrm{sec}, 4: 1$.
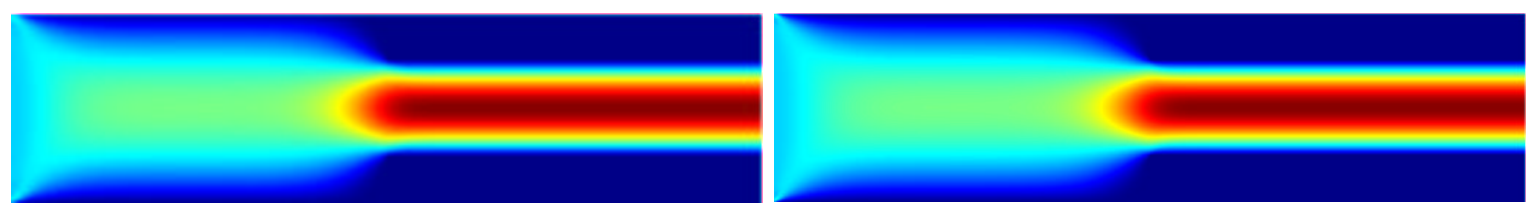

(c) Axial velocity, w, -0.007 (blue) to 3.0 (red) cm/sec, 2:1.
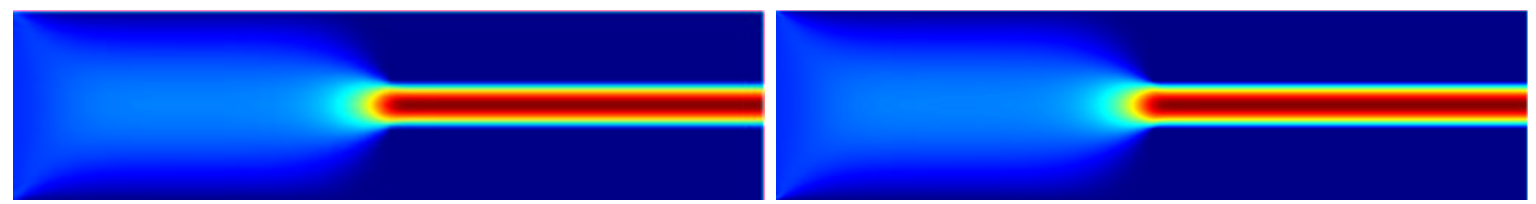

(d) Axial velocity, w, -0.02 (blue) to 6.0 (red) $\mathrm{cm} / \mathrm{sec}, 4: 1$.

Fig. 8. Convergence of viscous limit ( $W e=0$ ) of Oldroyd-B fluid at 2000 time steps. (L) $h=1 / 128 ;(\mathrm{R}) h=1 / 256$. 
List of Tables

Table I. Error and convergence rates for tranverse (left) and axial (right) velocity of Maxwell fluid, $M a=0.3, W e=205.6$ in 2:1 contraction channel at 2328 time steps $(h=1 / 256)$.

Table II. Error and convergence rates for tranverse (left) and axial (right) velocity of Maxwell fluid, $M a=0.6, W e=822.4$ in 4:1 contraction channel at 2554 time steps $(h=1 / 256)$.

Table III. Error and convergence rates for tranverse (left) and axial (right) velocity of Oldroyd-B fluid, $M a=0.06, W e=8$ in $2: 1$ contraction channel at 40 time steps $(h=1 / 256)$.

Table IV. Error and convergence rates for tranverse (left) and axial (right) velocity of Oldroyd-B fluid, $M a=0.12, W e=32$ in 4:1 contraction channel at 40 time steps $(h=1 / 256)$.

Table V. Error and convergence rates for tranverse (left) and axial (right) velocity of Oldroyd-B fluid, $M a=0.06, W e=8$ in 2:1 contraction channel at 1270 time steps $(h=1 / 256)$.

Table VI. Error and convergence rates for tranverse (left) and axial (right) velocity of Oldroyd-B fluid, $M a=0.12, W e=32$ in 4:1 contraction channel at 400 time steps $(h=1 / 256)$.

Table VII. Error and convergence rates for tranverse (left) and axial (right) velocity of Oldroyd-B fluid, $M a=0.12, W e=32$ in 4:1 contraction channel at 800 time steps $(h=1 / 256)$.

Table VIII. Error and convergence rates for tranverse (left) and axial (right) velocity of viscous limit $(W e=0)$ Oldroyd-B fluid in 2:1 contraction channel at 2000 time steps $(h=1 / 256)$.

Table IX. Error and convergence rates for tranverse (left) and axial (right) velocity of viscous limit $(W e=0)$ Oldroyd-B fluid in 4:1 contraction channel at 2000 time steps $(h=1 / 256)$. 
Table I

Error and convergence rates for tranverse (left) and axial (right) velocity of Maxwell fluid, $M a=0.3, W e=205.6$ in 2:1 contraction channel at 2328 time steps $(h=$ $1 / 256)$.

\begin{tabular}{lcccccc}
\hline \hline & & $\mathrm{u}$ & & \multicolumn{3}{c}{$\mathrm{w}$} \\
Norm & $e^{1 / 64}$ & Rate & $e^{1 / 128}$ & $e^{1 / 64}$ & Rate & $e^{1 / 128}$ \\
\hline \hline$L_{1}$ & $5.24 \times 10^{-3}$ & 1.78 & $1.54 \times 10^{-3}$ & $6.66 \times 10^{-1}$ & 2.16 & $1.49 \times 10^{-1}$ \\
$L_{2}$ & $5.28 \times 10^{-3}$ & 1.42 & $1.98 \times 10^{-3}$ & $4.90 \times 10^{-1}$ & 1.47 & $1.77 \times 10^{-1}$ \\
$L_{\infty}$ & $1.47 \times 10^{-2}$ & 0.52 & $1.03 \times 10^{-2}$ & $9.65 \times 10^{-1}$ & 0.52 & $6.75 \times 10^{-1}$ \\
\hline \hline
\end{tabular}


Table II

Error and convergence rates for tranverse (left) and axial (right) velocity of Maxwell fluid, $M a=0.6, W e=822.4$ in 4:1 contraction channel at 2554 time steps $(h=$ $1 / 256)$.

\begin{tabular}{lcccccc}
\hline \hline & & $\mathrm{u}$ & & $\mathrm{w}$ & \\
Norm & $e^{1 / 64}$ & Rate & $e^{1 / 128}$ & $e^{1 / 64}$ & Rate & $e^{1 / 128}$ \\
\hline \hline$L_{1}$ & $6.44 \times 10^{-3}$ & 2.06 & $1.54 \times 10^{-3}$ & $5.69 \times 10^{-1}$ & 2.40 & $1.08 \times 10^{-1}$ \\
$L_{2}$ & $7.19 \times 10^{-3}$ & 1.56 & $2.44 \times 10^{-3}$ & $5.36 \times 10^{-1}$ & 1.75 & $1.59 \times 10^{-1}$ \\
$L_{\infty}$ & $2.72 \times 10^{-2}$ & 0.97 & $1.39 \times 10^{-2}$ & 1.65 & 0.84 & $9.19 \times 10^{-1}$ \\
\hline \hline
\end{tabular}


Table III

Error and convergence rates for tranverse (left) and axial (right) velocity of OldroydB fluid, $M a=0.06, W e=8$ in $2: 1$ contraction channel at 40 time steps $(h=1 / 256)$.

\begin{tabular}{lcccccc}
\hline \hline & & $\mathrm{u}$ & & \multicolumn{3}{c}{$\mathrm{w}$} \\
Norm & $e^{1 / 64}$ & Rate & $e^{1 / 128}$ & $e^{1 / 64}$ & Rate & $e^{1 / 128}$ \\
\hline \hline$L_{1}$ & $2.39 \times 10^{-4}$ & 1.13 & $1.09 \times 10^{-4}$ & $3.89 \times 10^{-2}$ & 1.39 & $1.48 \times 10^{-2}$ \\
$L_{2}$ & $8.48 \times 10^{-4}$ & 1.17 & $3.77 \times 10^{-4}$ & $4.16 \times 10^{-2}$ & 1.39 & $1.59 \times 10^{-2}$ \\
$L_{\infty}$ & $1.72 \times 10^{-2}$ & 0.73 & $1.04 \times 10^{-2}$ & $2.01 \times 10^{-1}$ & 0.81 & $1.14 \times 10^{-1}$ \\
\hline \hline
\end{tabular}


Table IV

Error and convergence rates for tranverse (left) and axial (right) velocity of OldroydB fluid, $M a=0.12, W e=32$ in $4: 1$ contraction channel at 40 time steps $(h=$ $1 / 256)$.

\begin{tabular}{lcccccc}
\hline \hline & & $\mathrm{u}$ & & \multicolumn{3}{c}{$\mathrm{w}$} \\
Norm & $e^{1 / 64}$ & Rate & $e^{1 / 128}$ & $e^{1 / 64}$ & Rate & $e^{1 / 128}$ \\
\hline \hline$L_{1}$ & $4.05 \times 10^{-4}$ & 1.19 & $1.78 \times 10^{-4}$ & $6.28 \times 10^{-2}$ & 1.40 & $2.38 \times 10^{-2}$ \\
$L_{2}$ & $1.52 \times 10^{-3}$ & 1.19 & $6.69 \times 10^{-4}$ & $8.37 \times 10^{-2}$ & 1.39 & $3.20 \times 10^{-2}$ \\
$L_{\infty}$ & $3.06 \times 10^{-2}$ & 0.68 & $1.91 \times 10^{-2}$ & $3.71 \times 10^{-1}$ & 0.78 & $2.16 \times 10^{-1}$ \\
\hline \hline
\end{tabular}


Table V

Error and convergence rates for tranverse (left) and axial (right) velocity of OldroydB fluid, $M a=0.06, W e=8$ in $2: 1$ contraction channel at 1270 time steps $(h=$ $1 / 256)$.

\begin{tabular}{lcccccc}
\hline \hline & & $\mathrm{u}$ & & \multicolumn{3}{c}{$\mathrm{w}$} \\
Norm & $e^{1 / 64}$ & Rate & $e^{1 / 128}$ & $e^{1 / 64}$ & Rate & $e^{1 / 128}$ \\
\hline \hline$L_{1}$ & $3.44 \times 10^{-4}$ & 1.10 & $1.61 \times 10^{-4}$ & $5.66 \times 10^{-2}$ & 1.47 & $2.05 \times 10^{-2}$ \\
$L_{2}$ & $9.23 \times 10^{-4}$ & 1.12 & $4.26 \times 10^{-4}$ & $5.17 \times 10^{-2}$ & 1.51 & $1.82 \times 10^{-2}$ \\
$L_{\infty}$ & $1.19 \times 10^{-2}$ & 0.64 & $7.63 \times 10^{-3}$ & $1.55 \times 10^{-1}$ & 0.84 & $8.63 \times 10^{-2}$ \\
\hline \hline
\end{tabular}


Table VI

Error and convergence rates for tranverse (left) and axial (right) velocity of OldroydB fluid, $M a=0.12, W e=32$ in $4: 1$ contraction channel at 400 time steps $(h=$ $1 / 256)$.

\begin{tabular}{lcccccc}
\hline \hline & & $\mathrm{u}$ & & \multicolumn{3}{c}{$\mathrm{w}$} \\
Norm & $e^{1 / 64}$ & Rate & $e^{1 / 128}$ & $e^{1 / 64}$ & Rate & $e^{1 / 128}$ \\
\hline \hline$L_{1}$ & $4.56 \times 10^{-3}$ & 1.30 & $1.85 \times 10^{-3}$ & $5.26 \times 10^{-1}$ & 1.32 & $2.10 \times 10^{-1}$ \\
$L_{2}$ & $7.37 \times 10^{-3}$ & 1.12 & $3.40 \times 10^{-3}$ & $6.63 \times 10^{-1}$ & 1.22 & $2.84 \times 10^{-1}$ \\
$L_{\infty}$ & $4.07 \times 10^{-2}$ & 0.44 & $3.01 \times 10^{-2}$ & $1.63 \times 10^{-1}$ & 0.63 & $1.06 \times 10^{-1}$ \\
\hline \hline
\end{tabular}


Table VII

Error and convergence rates for tranverse (left) and axial (right) velocity of OldroydB fluid, $M a=0.12, W e=32$ in $4: 1$ contraction channel at 800 time steps $(h=$ $1 / 256)$.

\begin{tabular}{lcccccc}
\hline \hline & & $\mathrm{u}$ & & $\mathrm{w}$ & \\
Norm & $e^{1 / 64}$ & Rate & $e^{1 / 128}$ & $e^{1 / 64}$ & Rate & $e^{1 / 128}$ \\
\hline \hline$L_{1}$ & $5.92 \times 10^{-3}$ & 0.39 & $4.53 \times 10^{-3}$ & $5.46 \times 10^{-1}$ & 1.00 & $2.72 \times 10^{-1}$ \\
$L_{2}$ & $6.67 \times 10^{-3}$ & 0.16 & $5.99 \times 10^{-3}$ & $7.33 \times 10^{-1}$ & 1.06 & $3.50 \times 10^{-1}$ \\
$L_{\infty}$ & $2.72 \times 10^{-2}$ & 0.10 & $2.53 \times 10^{-2}$ & 1.70 & 0.20 & 1.48 \\
\hline \hline
\end{tabular}


Table VIII

Error and convergence rates for tranverse (left) and axial (right) velocity of viscous limit $(W e=0)$ Oldroyd-B fluid in 2:1 contraction channel at 2000 time steps $(h=$ $1 / 256)$.

\begin{tabular}{lcccccc}
\hline \hline & \multicolumn{3}{c}{$\mathrm{u}$} & \multicolumn{3}{c}{$\mathrm{w}$} \\
Norm & $e^{1 / 64}$ & Rate & $e^{1 / 128}$ & $e^{1 / 64}$ & Rate & $e^{1 / 128}$ \\
\hline \hline$L_{1}$ & $5.43 \times 10^{-5}$ & 1.04 & $2.64 \times 10^{-5}$ & $6.40 \times 10^{-3}$ & 1.18 & $2.83 \times 10^{-3}$ \\
$L_{2}$ & $1.03 \times 10^{-4}$ & 0.90 & $5.51 \times 10^{-5}$ & $9.96 \times 10^{-3}$ & 0.99 & $5.00 \times 10^{-3}$ \\
$L_{\infty}$ & $1.32 \times 10^{-3}$ & 1.23 & $5.62 \times 10^{-4}$ & $1.17 \times 10^{-1}$ & 1.28 & $4.83 \times 10^{-2}$ \\
\hline \hline
\end{tabular}


Table IX

Error and convergence rates for tranverse (left) and axial (right) velocity of viscous limit $(W e=0)$ Oldroyd-B fluid in 4:1 contraction channel at 2000 time steps $(h=$ $1 / 256)$.

\begin{tabular}{lcccccc}
\hline \hline & \multicolumn{3}{c}{$\mathrm{u}$} & \multicolumn{3}{c}{$\mathrm{w}$} \\
Norm & $e^{1 / 64}$ & Rate & $e^{1 / 128}$ & $e^{1 / 64}$ & Rate & $e^{1 / 128}$ \\
\hline \hline$L_{1}$ & $6.36 \times 10^{-5}$ & 0.96 & $3.27 \times 10^{-5}$ & $1.06 \times 10^{-2}$ & 1.31 & $4.29 \times 10^{-3}$ \\
$L_{2}$ & $1.89 \times 10^{-4}$ & 0.82 & $1.07 \times 10^{-4}$ & $2.15 \times 10^{-2}$ & 1.02 & $1.06 \times 10^{-2}$ \\
$L_{\infty}$ & $3.32 \times 10^{-3}$ & 0.45 & $2.44 \times 10^{-3}$ & $3.08 \times 10^{-1}$ & 0.50 & $2.18 \times 10^{-1}$ \\
\hline \hline
\end{tabular}

